\title{
PAPER \\ Randomization Approaches for Reducing PAPR with Partial Transmit Sequence and Semidefinite Relaxation
}

\author{
Hirofumi TSUDA $^{\dagger \mathrm{a})}$, Nonmember and Ken UMENO ${ }^{\dagger \dagger \mathrm{b})}$, Member
}

\begin{abstract}
SUMMARY To reduce peak-to-average power ratio, we propose a method of choosing suitable vectors in a partial transmit sequence technique. Conventional approaches require that a suitable vector be selected from a large number of candidates. By contrast, our method does not include such a selecting procedure, and instead generates random vectors from the Gaussian distribution whose covariance matrix is a solution of a relaxed problem. The suitable vector is chosen from the random vectors. This yields lower peak-to-average power ratio than a conventional method. key words: orthogonal frequency division multiplexing (OFDM), peak-toaverage power ratio (PAPR), partial transmit sequence, semidefinite relaxation, randomization algorithm
\end{abstract}

\section{Introduction}

Orthogonal Frequency Division Multiplexing (OFDM) systems are widely used and their signals are generated by Inverse Fast Fourier Transformation (IFFT) [1]. One advantage for OFDM systems is that OFDM systems can deal with multi-path delay since OFDM systems are implemented with IFFT, and effects of multi-path delay in frequency selective channels can be removed with guard interval techniques and zero padding techniques [2]. Since OFDM systems can remove the effects of multi-path delay, Multiple-Input Multiple-Output (MIMO) systems with OFDM systems have been investigated [3].

While there are some advantages in OFDM systems, there are two main problems. One is that signals of OFDM systems have relatively large side-lobes [4]. This problem is caused since OFDM signals consists of sine waves. The other is that signals of OFDM systems have large Peakto-Average Power Ratio (PAPR), which is the ratio of the maximum value of RF signal powers to the average value of them. Approximately, the output power grows linearly for low values of the input powers. However, for input signals with large power, the growth of the output power is nonlinear. Then, in-band distortion and out-of-band distortion are caused for a large input power [5]. With symbols chosen independently, PAPR for OFDM signals has been investigated in [6], [7]. Further, in such a case, it has been proven that a baseband-signal can be regarded as a Gaussian process

Manuscript received November 25, 2019.

Manuscript revised July 20, 2020.

Manuscript publicized September 1, 2020.

${ }^{\dagger}$ The author is with T\&S inc., Yokohama-shi, 220-0012 Japan.

${ }^{\dagger}$ The author is with the Kyoto University, Kyoto-shi, 606-8501 Japan.

a) E-mail: tsuda.hirofumi.38u@kyoto-u.jp

b) E-mail: umeno.ken.8z@kyoto-u.ac.jp

DOI: 10.1587/transfun.2019EBP3243
[8]. With dependent symbols, for example, Bose-ChaudhuriHocquenghem $(\mathrm{BCH})$ codes, their PAPR has been investigated [9]. Further, the performance of OFDM systems with non-linear amplifiers has been investigated in [10], [11].

To reduce PAPR, many methods have been proposed and explored. For example, a selected mapping method [12], a balancing method [13], an active constellation extension method [14], a tone injection method [15], a complement block coding method [16], a constellation reshaping method [17], an iterative filtering method [18], and a compounding method [19]. These methods are summarized in [20]-[22].

One of the methods to reduce PAPR is a Partial Transmit Sequence (PTS) technique [23]-[26]. PTS techniques are to multiply symbols by phases to reduce PAPR. It is necessary to transmit the vector as side information to the receiver. This yields that OFDM signals are not distorted since we only modulate symbols. Then, their side lobes stay unchanged.

With PTS techniques, there is a significant task for reducing PAPR, how to reduce the calculation amount. In [23], a suitable vector is chosen as one which achieves the lowest PAPR from all of the candidates. Then, the calculation amount exponentially gets larger as the length of a vector increases. To reduce such calculations, some methods have been known. In [27], a neighborhood search algorithm has been proposed. With this method, we can obtain a local optimal solution. Another method is a phase random method [28]. This method consists of generating random vectors whose phase is uniformly distributed in the set of the candidates.

In this paper, we propose a method to search the vector which achieves low PAPR. The main point of our method is to obtain the vector from a set of random vectors generated from the Gaussian distribution. Therefore, our method is similar to the phase random method. Then, we derive the optimization problem to reduce PAPR and obtain a solution from the relaxed problem. We regard the solution as a covariance matrix and we can determine the Gaussian distribution.

Our method is a probabilistic scheme since the vectors are generated from a Gaussian distribution. There are some probabilistic schemes in PAPR reduction techniques. A selective mapping method was proposed in [12] to generate phases randomly to obtain independent vectors. In [29], for a PTS technique, it has been proposed to choose sub-blocks randomly. Therefore, our method is one such scheme.

The rest of this paper is organized as follows: Section 2 shows the definitions of PAPR and Peak-to-Mean Envelope Power Ratio (PMEPR). In the literature, these two notions 
sometimes are assumed to coincide, however, these two are different since PAPR and PMEPR are defined with RF signals and base-band signals, respectively. In this section, we clarify the property of signals considered in this paper. Section 3 shows a partial transmit sequence technique and an optimization problem. It is not straightforward to solve this optimization problem since the feasible region of this optimization problem is discrete. Therefore, in Sect. 4, we show a semidefinite relaxation technique to solve it. With this technique, we can obtain approximate solutions. In Sect. 5, we consider random vectors generated from the Gaussian distribution whose covariance matrix is the solution of the relaxed problem. Then, in Sect. 6, we show the relation between our randomization method and the phase random method, which is a conventional method. In Sect. 7, since our problem stated in Sects. 4 and 5 has the large number of constraints, we propose another optimization problem to reduce the upper bound of PAPR. This problem has fewer constraints than the problems in Sects. 4 and 5. Finally, we compare the PAPR of our method with one of existing methods.

The two conference papers by the present authors [25], [26] have shown the randomization methods with certain parameters, which is summarized in this paper. The new contribution of this paper is that more general methods are proposed. We obtain the optimization problem with each kind of parameters and clarify the relation between our methods and the phase random method, which is described in Sect. 6. Further, we consider the upper bound of PAPR and obtain the optimization problem, which is described in Sect. 7. In Sect. 8, we investigate the Bit Error Rate performances with our methods and existing ones.

\section{OFDM System Model and PAPR}

In this section, we fix the model and the quantities used throughout this paper. A complex baseband OFDM signal is written as [1]

$$
s(t)=\sum_{k=1}^{K} A_{k} \exp \left(2 \pi j \frac{k-1}{T} t\right), \quad 0 \leq t<T,
$$

where $A_{k}$ is a $k$-th transmitted symbol, $K$ is the number of symbols, $j$ is the unit imaginary number, and $T$ is a duration of symbols. It is known that OFDM signals are generated by Inverse Fast Fourier Transformation (IFFT) [1]. As seen in Eq. (1), the OFDM signals have no cyclic prefixes. With the cyclic prefix technique, the PAPR is preserved since the cyclic prefix does not induce any new peaks [32]. Therefore, we consider such OFDM signals written in Eq. (1).

A Radio Frequency (RF) OFDM signal $\zeta(t)$ is written with Eq. (1) as

$$
\begin{aligned}
\zeta(t) & =\operatorname{Re}\left\{s(t) \exp \left(2 \pi j f_{c} t\right)\right\} \\
& =\operatorname{Re}\left\{\sum_{k=1}^{K} A_{k} \exp \left(2 \pi j\left(\frac{k-1}{T}+f_{c}\right) t\right)\right\},
\end{aligned}
$$

where $\operatorname{Re}\{z\}$ is the real part of $z$, and $f_{c}$ is a carrier frequency. With RF signals, PAPR is defined as [30], [31]

$$
\mathrm{PAPR}=\max _{0 \leq t<T} \frac{\left|\operatorname{Re}\left\{\sum_{k=1}^{K} A_{k} \exp \left(2 \pi j\left(\frac{k-1}{T}+f_{c}\right) t\right)\right\}\right|^{2}}{P_{\mathrm{av}}},
$$

where $P_{\mathrm{av}}$ corresponds to the average power of signals, $P_{\mathrm{av}}=$ $\sum_{k=1}^{K} \mathrm{E}\left\{\left|A_{k}\right|^{2}\right\}$, and $\mathrm{E}\{X\}$ is the average of $X$. Similarly, with baseband signals, PMEPR is defined as [30], [31]

$$
\text { PMEPR }=\max _{0 \leq t<T} \frac{\left|\sum_{k=1}^{K} A_{k} \exp \left(2 \pi j \frac{k-1}{T} t\right)\right|^{2}}{P_{\mathrm{av}}} .
$$

From Eqs. (3) and (4), PAPR and PMEPR are determined if the transmitted symbols $\left\{A_{k}\right\}$ are given. In the literature, PAPR and PMEPR have often been evaluated as probabilities, since PAPR and PMEPR depend on symbols $A_{k}$ that can be regarded as random variables [6], [9].

Obviously, PAPR does not always correspond to PMEPR. Further, from Eqs. (3) and (4), PAPR does not exceed PMEPR. In [32], under some conditions described below, it has been proven that the following relations are established

$$
\left(1-\frac{\pi^{2} K^{2}}{2 r^{2}}\right) \cdot \text { PMEPR } \leq \text { PAPR } \leq \text { PMEPR, }
$$

where $r$ is an integer such that $f_{c}=r / T$. The conditions that Eq. (5) holds are $K \ll r$ and $\exp (2 \pi j K / r) \approx 1$. In addition to these, another relation has been shown in [21]. Equation (5) implies that PMEPR approximately equals PAPR for sufficiently large $f_{c}$. It is often the case that PMEPR is evaluated instead of PAPR [6]. In what follows, we assume that the carrier frequency $f_{c}$ is sufficiently large, that is, we consider baseband OFDM signals instead of RF signals.

\section{Partial Transmit Sequence Technique}

With OFDM systems, the Partial Transmit Sequence (PTS) technique has been proposed to reduce PAPR. In this section, we show the model and the details of PTS techniques. PTS techniques need a vector to reduce PAPR. A disadvantage of PTS techniques is that a large amount of calculation is necessary in some situations. The details of PTS techniques are described in [21], [23], [33].

The symbols in the paper and how to derive index sets for symbols are given as follows. We assume that the symbols $A_{k}$ are given and the number of the symbols is $K$. Further, the index set $\Lambda=\{1,2, \ldots, K\}$ corresponds to the set of unordered symbols $\left\{A_{1}, A_{2}, \ldots, A_{K}\right\}$. To apply a PTS technique, we divide the index set $\Lambda$ into $P$ disjoint subsets, $\Lambda_{1}, \ldots, \Lambda_{P}$, that is,

$$
\Lambda=\Lambda_{1} \cup \cdots \cup \Lambda_{P}, \quad \Lambda_{k} \cap \Lambda_{m}=\emptyset \text { if } k \neq m
$$


for $k, m=1,2, \ldots, P$. There are some discussions about how to divide the index set. We refer the reader to [29], [34], [35].

To express the instantaneous power, we define some quantities as below. For each subsets of symbols, we introduce a rotation vector $\mathbf{b}=\left(b_{1}, b_{2}, \ldots, b_{P}\right)^{\top}$, where $\mathbf{x}^{\top}$ is the transpose of $\mathbf{x}$. The vector $\mathbf{b}$ is chosen as one satisfying $b_{p}=\exp \left(j \theta_{p}\right)$ for $p=1,2, \ldots, P$, where $\theta_{p} \in[0,2 \pi)$. This b plays various roles throughout this paper. For convenience, let us define the quantities

$$
A_{k}^{(p)}=\left\{\begin{array}{cc}
A_{k} & k \in \Lambda_{p} \\
0 & \text { otherwise }
\end{array}\right.
$$

for $k=1,2, \ldots, K$ and $p=1,2, \ldots, P$. With $A_{k}^{(p)}$, a modified baseband OFDM signal $\hat{s}(t)$ is written as

$$
\hat{s}(t)=\sum_{p=1}^{P} \sum_{k=1}^{K} A_{k}^{(p)} b_{p} \exp \left(2 \pi j \frac{k-1}{T} t\right) .
$$

Note that the average power of modified signals is equivalent to one of the original OFDM signals since $\left|b_{p}\right|=1$. With a matrix and vectors, the above equation is rewritten as

$$
\hat{s}(t)=\mathbf{v}_{t}^{\top} A \mathbf{b}
$$

where

$$
\begin{aligned}
& \mathbf{v}_{t}=\left(\begin{array}{cccc}
v_{1, t} & v_{2, t} & \cdots & v_{K, t}
\end{array}\right)^{\top}, \\
& A=\left(\begin{array}{cccc}
A_{1}^{(1)} & A_{1}^{(2)} & \cdots & A_{1}^{(P)} \\
A_{2}^{(1)} & A_{2}^{(2)} & \cdots & A_{2}^{(P)} \\
\vdots & \vdots & \ddots & \vdots \\
A_{K}^{(1)} & A_{K}^{(2)} & \cdots & A_{K}^{(P)}
\end{array}\right)
\end{aligned}
$$

and

$$
v_{k, t}=\exp \left(2 \pi j \frac{k-1}{T} t\right) .
$$

Note that the matrix $A$ is a $K \times P$ matrix. With these quantities, the instantaneous power $|\hat{s}(t)|^{2}$ is written as

$$
|\hat{s}(t)|^{2}=\mathbf{b}^{*} A^{*}\left(\mathbf{v}_{t}^{*}\right)^{\top} \mathbf{v}_{t}^{\top} A \mathbf{b},
$$

where $\mathbf{z}^{*}$ and $X^{*}$ are the complex conjugate transposes of $\mathbf{z}$ and $X$, respectively. Here, we have used the fact $\left(\mathbf{v}^{\top}\right)^{*}=$ $\left(\mathbf{v}^{*}\right)^{\top}$. We denote by $C_{t}$ the matrix $A^{*}\left(\mathbf{v}_{t}^{*}\right)^{\top} \mathbf{v}_{t}^{\top} A$. Note that $C_{t}$ is a positive semidefinite matrix since $C_{t}$ is a Gram matrix and each value of $b_{p}$ is chosen as one achieving the lowest PAPR.

At the receiver side, to recover symbols, it is necessary for the receiver to know the explicit values of $\mathbf{b}$. Note that Signal to Noise Ratio (SNR) is preserved if the receiver knows b. To let the receiver know, the vector $\mathbf{b}$ has to be transmitted as side-information. To reduce information content, the value of $b_{p}$ is usually restricted to

$$
b_{p} \in\left\{1, \exp \left(2 \pi j \frac{1}{L}\right), \ldots, \exp \left(2 \pi j \frac{L-1}{L}\right)\right\},
$$

where $L$ is a positive integer. We denote by $\Omega_{L}$ the set $\left\{1, \exp \left(2 \pi j \frac{1}{L}\right), \ldots, \exp \left(2 \pi j \frac{L-1}{L}\right)\right\}$. From the above definition, the vector $\mathbf{b}$ belongs $\Omega_{L}^{P}$, where $\Omega_{L}^{P}$ is a $P$-tuple of $\Omega_{L}$. Then, the information content of $\mathbf{b}$ is $(P-1) \log _{2} L$ [bits] since we can set $b_{1}=1$ without loss of generality. It is obvious that the number of elements in the set $\Omega_{L}^{P-1}$ is $L^{P-1}$. Let $\mathbf{b}^{\star}$ be the optimal vector which achieves the lowest PAPR in $\Omega_{L}^{P}$. Then, it turns out that $\mathbf{b}^{\star}$ is the global solution of the optimization problem

$$
\begin{array}{cc}
\left(Q_{L}\right) \quad \min \max _{0 \leq t<T}|\hat{s}(t)|^{2} \\
\text { subject to } \quad b_{p} \in \Omega_{L} \quad(p=1,2, \ldots, P) .
\end{array}
$$

Our aim is to find the vector $\mathbf{b}^{\star}$. To this end, there are two main obstacles to solve the problem $\left(Q_{L}\right)$.

One obstacle is that the time $t$ is continuous. In [32], with baseband OFDM signals $s(t)$ defined in Eq. (1), it has been shown that there is the following relation between continuous signals and sampled signals

$$
\max _{0 \leq t<T}|s(t)|<\sqrt{\frac{J^{2}}{J^{2}-\pi^{2} / 2}} \max _{0 \leq n<J K}\left|s\left(\frac{n T}{J K}\right)\right|,
$$

where $J$ is an integer satisfying $J>\pi / \sqrt{2}$. Equation (15) implies that PMEPR can be estimated precisely from signals sampled with a sufficiently large oversampling factor. For maxima of continuous signals and sampled signals, other relations have been shown in [36], [37]. The integer $J$ is often called a oversampling factor [10], and is often chosen as $J \geq 4$. How to choose the oversampling factor $J$ has been discussed in [32].

With sampled signals, the problem $\left(Q_{L}\right)$ is rewritten as

$\left(\hat{Q}_{L}\right) \quad \min \lambda$

$$
\begin{array}{ll}
\text { subject to } & \mathbf{b}^{*} C_{n T /(J K)} \mathbf{b} \leq \lambda \quad(n=0,1, \ldots, J K-1) \\
& \lambda \in \mathbb{R}, \quad b_{p} \in \Omega_{L} \quad(p=1,2, \ldots, P) .
\end{array}
$$

Note that the variables in the problem $\left(\hat{Q}_{L}\right)$ are $\mathbf{b}$ and $\lambda$.

The other obstacle is that the feasible region $\Omega_{L}$ is discrete. In [23], a brute-force search has been used to find the global solution $\mathbf{b}^{\star}$. With this method, we have to find the vector $\mathbf{b}^{\star}$ from $L^{P-1}$ candidates, and the calculation amount exponentially gets larger as $P$ increases. In [27], the neighborhood search algorithm has been proposed. With this method, we can obtain a local optimal solution. However, it is only known that its calculation amount is proportional to ${ }_{P-1} C_{r} \cdot L^{r}$, where $r$ is an integer parameter expressing the distance of a neighborhood and ${ }_{a} C_{b}$ is a binomial coefficient. Another existing method is the phase random method [28]. This method consists of generating random vectors whose phase is uniformly distributed in the region $\Omega_{L}^{P}$, from which we obtain a solution.

\section{Semidefinite Relaxation}

Since it is not straightforward to obtain the global solu- 
tion, we propose an efficient method to obtain a solution which achieves low PAPR. Optimization problems, such as the problem $\left(\hat{Q}_{L}\right)$, appear in MIMO detection [38]. Thus, we can use these methods that have already been developed to our problem. One of such existing methods uses a semidefinite relaxation technique [39]. In this section, we obtain a solution with such semidefinite relaxation techniques.

We apply semidefinite relaxation techniques to the problem $\left(\hat{Q}_{L}\right)$. Our main aim is to change the variable $\mathbf{b}$ to a positive semidefinite matrix $X$. The ways to solve the problem $\left(\hat{Q}_{L}\right)$ depend on $\Omega_{L}$. Therefore, we consider each problem for various cases of $L$.

\subsection{Optimization Problem for $L=2$}

First, we consider the problem $\left(\hat{Q}_{L}\right)$ for $L=2,\left(\hat{Q}_{2}\right)$. Then, $\Omega_{2}=\{-1,1\}$. Note that $b^{2}=1$ for $b \in \Omega_{2}$. If we define the matrix $X=\mathbf{b b}^{\top}$, then $X$ is a positive semidefinite matrix whose rank is 1 and the problem $\left(\hat{Q}_{2}\right)$ is rewritten as

\section{$\left(\hat{Q}_{2}\right) \quad \min \lambda$}

subject to $\operatorname{Tr}\left(C_{n T /(J K)} X\right) \leq \lambda \quad(n=0,1, \ldots, J K-1)$

$$
\operatorname{rank}(X)=1, \quad X_{p, p}=1 \quad(p=1,2, \ldots, P)
$$$$
X \geqslant 0, \quad X \in \mathbb{S}_{P}, \quad \lambda \in \mathbb{R},
$$

where $\operatorname{Tr}(X)$ is the trace of $X, \operatorname{rank}(X)$ is the rank of $X$, $X \geqslant 0$ indicates that $X$ is a positive semidefinite matrix and $\mathbb{S}_{P}$ is the set of symmetric matrices of dimension $P$. Due to the constraint $\operatorname{rank}(X)=1$, the problem $\left(\hat{Q}_{2}\right)$ is not convex. Note that the set of positive semidefinite matrices is convex [40]. By dropping the rank constraint, we obtain the relaxed optimization problem

$$
\left(\hat{Q}_{2}^{\prime}\right) \quad \min \lambda
$$

subject to $\operatorname{Tr}\left(C_{n T /(J K)} X\right) \leq \lambda \quad(n=0,1, \ldots, J K-1)$

$$
X_{p, p}=1 \quad(p=1,2, \ldots, P)
$$$$
X \geqslant 0, \quad X \in \mathbb{S}_{P}, \quad \lambda \in \mathbb{R} \text {. }
$$

The above problem $\left(\hat{Q}_{2}^{\prime}\right)$ can be solved since the problem $\left(\hat{Q}_{2}^{\prime}\right)$ is convex. Let $X_{2}^{\star}$ be the global solution of the problem $\left(\hat{Q}_{2}^{\prime}\right)$. If the rank of $X_{2}^{\star}$ is 1 , then we obtain the global solution of the problem $\left(\hat{Q}_{2}\right)$, denoted by $\mathbf{b}_{2}^{\star}$. However, the rank of $X_{2}^{\star}$ is not always 1 . To deal with this, we obtain an approximate solution from $X_{2}^{\star}$ as follows. First, the solution $X_{2}^{\star}$ is decomposed as

$$
X_{2}^{\star}=\sum_{i=1}^{r_{2}} \lambda_{i} \mathbf{q}_{i} \mathbf{q}_{i}^{*}
$$

where $r_{2}=\operatorname{rank}\left(X_{2}^{\star}\right), \lambda_{i}$ is the eigenvalue of $X_{2}^{\star}, \lambda_{1} \geq$ $\lambda_{2} \geq \ldots \geq \lambda_{r_{2}}$ and $\mathbf{q}_{i}$ is the respective eigenvector. Then, in a least-two-norm sense, the approximate solution whose rank 1 is obtained as $\hat{X}_{2}^{\star}=\lambda_{1} \mathbf{q}_{1} \mathbf{q}_{1}^{*}$. From this approximate solution, we systematically obtain the solution of the original problem $\left(\hat{Q}_{2}\right)$ as $\sqrt{\lambda_{1}} \mathbf{q}_{1}$. However, this solution is not always in the feasible region of the problem $\left(\hat{Q}_{2}\right)$. To have an approximate solution in the feasible region, for the problem $\left(\hat{Q}_{2}\right)$, we need to project the solution onto the feasible region. We finally arrive at the $p$-th element of the approximate solution of the problem $\left(\hat{Q}_{2}\right)$ as

$$
\hat{b}_{2, p}=\operatorname{sgn}\left(q_{1, p}\right),
$$

where $q_{1, p}$ is the $p$-th element of $\mathbf{q}_{1}$ and

$$
\operatorname{sgn}(x)=\left\{\begin{array}{cc}
1 & x \geq 0 \\
-1 & x<0
\end{array} .\right.
$$

We call this technique " $l_{2}$ approximation method".

\subsection{Optimization Problem for $L=4$}

Similar to the case $L=2$, we obtain the approximate solution of the problem $\left(\hat{Q}_{4}\right)$. For $L=4$, the set $\Omega_{4}$ is written as $\Omega_{4}=\{1, \exp (j \pi / 2),-1, \exp (j 3 \pi / 4)\}$. To obtain the relaxed problem, we rewrite the problem $\left(\hat{Q}_{4}\right)$ as follows. First, let us define the set

$$
\hat{\Omega}_{4}=\{+1+j,+1-j,-1+j,-1-j\},
$$

which can be expressed as

$$
\hat{\Omega}_{4}=\left\{\sqrt{2} \exp (j \pi / 4) \cdot a \mid a \in \Omega_{4}\right\} .
$$

Note that $\operatorname{Re}\{b\}^{2}=\operatorname{Im}\{b\}^{2}=1$ for $b \in \hat{\Omega}_{4}$ and that the elements in $\hat{\Omega}_{4}$ are complex. Second, we rewrite the set $\hat{\Omega}_{4}$ in terms of real parts and imaginary parts. To this end, we introduce the following transformations for $\mathbf{z} \in \mathbb{C}^{n}$ and $Z \in$ $\mathbb{H}_{n}$, where $\mathbb{H}_{n}$ is the set of Hermitian matrices of dimension $n[41]$,

$$
\mathcal{T}(\mathbf{z})=\left(\begin{array}{c}
\operatorname{Re}\{\mathbf{z}\} \\
\operatorname{Im}\{\mathbf{z}\}
\end{array}\right), \quad \text { and } \mathcal{T}(Z)=\left(\begin{array}{cc}
\operatorname{Re}\{Z\} & -\operatorname{Im}\{Z\} \\
\operatorname{Im}\{Z\} & \operatorname{Re}\{Z\}
\end{array}\right) .
$$

Note that $\mathcal{T}(X) \in \mathbb{S}_{2 n}$ if $X \in \mathbb{H}_{n}$ [42]. Finally, we arrive at the relaxed problem for $L=4$.

$$
\begin{aligned}
&\left(\hat{Q}_{4}^{\prime}\right) \quad \min \lambda \\
& \text { subject to } \operatorname{Tr}\left(\hat{C}_{n T /(J K)} X\right) \leq \lambda \quad(n=0,1, \ldots, J K-1) \\
& X_{p, p}=1 \quad(p=1,2, \ldots, 2 P) \\
& X \geqslant 0, \quad X \in \mathbb{S}_{2 P}, \quad \lambda \in \mathbb{R},
\end{aligned}
$$

where $\hat{C}_{t}=\mathcal{T}\left(C_{t}\right)$. Note that the problem $\left(\hat{Q}_{4}^{\prime}\right)$ is equivalent to $\left(\hat{Q}_{4}\right)$ if we impose the rank constraints to the problem $\left(\hat{Q}_{4}^{\prime}\right)$ and the problem $\left(\hat{Q}_{4}^{\prime}\right)$ is convex. Let $X_{4}^{\star}$ be the global solution of the problem $\left(\hat{Q}_{4}^{\prime}\right)$. From $X_{4}^{\star}$, we can obtain an approximate solution by the following procedure. First, we decompose $X_{4}^{\star}$ as

$$
X_{4}^{\star}=\sum_{i=1}^{r_{4}} \lambda_{i} \mathbf{q}_{i} \mathbf{q}_{i}^{*}
$$


where $r_{4}=\operatorname{rank}\left(X_{4}^{\star}\right), \lambda_{i}$ is the eigenvalue of $X_{4}^{\star}, \lambda_{1} \geq \lambda_{2} \geq$ $\ldots \geq \lambda_{r_{4}}$, and $\mathbf{q}_{i}$ is the respective eigenvector. From the vector $\mathbf{q}_{1}$, we can obtain the approximate solution $\hat{\mathbf{b}}_{4} \in \mathbb{C}^{P}$ written as

$$
\hat{b}_{4, p}=\frac{1}{\sqrt{2}} \exp (-j \pi / 4)\left(\operatorname{sgn}\left(q_{p}\right)+j \cdot \operatorname{sgn}\left(q_{p+P}\right)\right),
$$

where $\hat{b}_{4, p}$ and $q_{p}$ are the $p$-th elements of $\hat{\mathbf{b}}_{4}$ and $\mathbf{q}_{1}$, respectively.

\subsection{Optimization Problem for General $L$}

For general $L$, we consider the relaxed problem

$$
\begin{aligned}
\left(\hat{Q}_{L}^{\prime}\right) & \min \lambda \\
\text { subject to } & \operatorname{Tr}\left(C_{n T /(J K)} X\right) \leq \lambda \quad(n=0,1, \ldots, J K-1) \\
& X_{p, p}=1 \quad(p=1,2, \ldots, P) \\
& X \geqslant 0, \quad X \in \mathbb{H}_{P}, \quad \lambda \in \mathbb{R} .
\end{aligned}
$$

Note that the set of Hermitian positive semidefinite matrices is convex [39] and the above problem $\left(\hat{Q}_{L}^{\prime}\right)$ is convex. The problem $\left(\hat{Q}_{L}^{\prime}\right)$ is not equivalent to the problem $\left(\hat{Q}_{L}\right)$ for $L \neq 2,4$ if the rank constraint is imposed. Similar to the problem for $L=2$ and $L=4$, let $X_{L}^{\star}$ be the global solution of the problem $\left(\hat{Q}_{L}^{\prime}\right)$. Then, $X_{L}^{\star}$ is decomposed as

$$
X_{L}^{\star}=\sum_{i=1}^{r_{L}} \lambda_{i} \mathbf{q}_{i} \mathbf{q}_{i}^{*},
$$

where $r_{L}=\operatorname{rank}\left(X_{L}^{\star}\right), \lambda_{i}$ is the eigenvalue of $X_{L}^{\star}, \lambda_{1} \geq \lambda_{2} \geq$ $\ldots \geq \lambda_{r_{L}}$ and $\mathbf{q}_{i}$ is the respective eigenvector. From the vector $\mathbf{q}_{1}$, we can obtain the approximate solution $\hat{\mathbf{b}}_{L} \in \mathbb{C}^{P}$ as

$$
\hat{\mathbf{b}}_{L}=\underset{\mathbf{b} \in \Omega_{L}^{P}}{\arg \min }\|\mathbf{q}-\mathbf{b}\|,
$$

where $\mathbf{q}=\sqrt{\lambda_{1}} \mathbf{q}_{1}$ and $\|\mathbf{z}\|$ is the Euclidean norm of $\mathbf{z}$.

\section{Randomization Method}

In Sect. 4, we have discussed the relaxed problems and how to obtain the approximate solutions. However, clearly, approximate solutions are not suitable if the global solutions of the relaxed problems have some large eigenvalues, that is, the ranks of solutions are not regarded as unity.

In this section, we introduce a randomization method. This method has been used to analyze how far the optimal value of relaxed problems is from one of original problems [43]. With this method, we obtain solutions as random values which are generated from a Gaussian distribution. Similar to discussions in Sect. 4, we consider each problem for various cases of $L$. Further, in the end of this section, we discuss the computational complexity of our randomization method.

\subsection{Randomization for $L=2$}

First, we consider the problem for $L=2$. Let $\boldsymbol{\xi}$ be a random vector generated from the Gaussian distribution $\mathcal{N}(\mathbf{0}, X)$ with zero mean and a covariance matrix $X$. The definition and properties of a Gaussian distribution have been shown in [44].

To find an approximate solution, we rewrite the problem $\left(\hat{Q}_{2}^{\prime}\right)$ as follows. With

$$
\mathrm{E}\left\{\boldsymbol{\xi}^{\top} C_{t} \boldsymbol{\xi}\right\}=\operatorname{Tr}\left(C_{t} X\right),
$$

the problem $\left(\hat{Q}_{2}^{\prime}\right)$ can be written as

$$
\left(\hat{Q}_{2}^{\prime}\right) \quad \min \lambda
$$

$$
\begin{array}{ll}
\text { subject to } & \mathrm{E}\left\{\boldsymbol{\xi}^{\top} C_{n T /(J K)} \boldsymbol{\xi}\right\} \leq \lambda \quad(n=0,1, \ldots, J K-1) \\
& X_{p, p}=1 \quad(p=1,2, \ldots, P) \\
& X \geqslant 0, \quad X \in \mathbb{S}_{P}, \quad \lambda \in \mathbb{R}, \quad \boldsymbol{\xi} \sim \mathcal{N}(0, X) .
\end{array}
$$

Note that the variables of the above problem are $X$ and $\lambda$. Then, it is clear that the optimal matrix $X_{2}^{\star}$ defined in Sect. 4 is the optimal matrix of the above problem in a sense of a covariance matrix. This result suggests that a suitable solution can be obtained from a set of random vectors generated from the Gaussian distribution $\mathcal{N}\left(\mathbf{0}, X_{2}^{\star}\right)$ [45]. We can then obtain the approximate solution as follows.

1. Solve the problem $\left(\hat{Q}_{2}^{\prime}\right)$ and obtain the covariance ma$\operatorname{trix} X_{2}^{\star}$.

2. Generate random vectors $\{\xi\}$ from the Gaussian distribution $\mathcal{N}\left(\mathbf{0}, X_{2}^{\star}\right)$ and project them onto the feasible region of the original problem $\left(\hat{Q}_{L}\right)$, that is, for $L=2$, obtain the projected solutions

$$
\hat{b}_{p}=\operatorname{sgn}\left(\xi_{p}\right) \quad(p=1,2, \ldots, P),
$$

where $\xi_{p}$ is the $p$-th element of $\boldsymbol{\xi}$.

3. Choose the solution which achieves the minimum PAPR among all the random vectors and regard it as an approximate solution.

\subsection{Randomization for $L=4$}

Similar to the case for $L=2$, we can obtain the covariance matrix $X_{4}^{\star}$ for $L=4$ and obtain random vectors $\{\xi\}$ generated from $\mathcal{N}\left(\mathbf{0}, X_{4}^{\star}\right)$. Since the dimension of the vectors $\{\boldsymbol{\xi}\}$ is $2 P$, the projected one is written as

$$
\hat{b}_{p}=\frac{1}{\sqrt{2}} \exp (-j \pi / 4)\left(\operatorname{sgn}\left(\xi_{p}\right)+j \cdot \operatorname{sgn}\left(\xi_{p+P}\right)\right)
$$

for $p=1,2, \ldots, P$. From these random vectors, we choose an approximate solution which achieves the minimum PAPR among them. 


\subsection{Randomization with General $L$}

For general $L$, we can obtain the complex covariance matrix $X_{L}^{\star}$ as a solution of the problem $\left(\hat{Q}_{L}^{\prime}\right)$. Similar to the methods for $L=2$ and $L=4$, our goal is to choose the solution from random vectors. The main part of our method is to obtain an approximate solution from random vectors generated from the complex Gaussian distribution $C \mathcal{N}\left(\mathbf{0}, X_{L}^{\star}\right)$. The definition and the detail of a complex Gaussian distribution have been shown in [46]. There have been some methods to obtain an approximate solution from random vectors [47] [49], and our method is a special case of an algorithm in [49]. From the complex Gaussian distribution $C \mathcal{N}\left(\mathbf{0}, X_{L}^{\star}\right)$, we can obtain the random vectors $\{\boldsymbol{\xi}\}$. Then, we have to transform the random vectors $\{\boldsymbol{\xi}\}$ into feasible ones as solutions of the problem $\left(\hat{Q}_{L}\right)$. Our transformation method is written as follows. Let $f_{L}$ be

$$
f_{L}(z)=\left\{\begin{array}{cl}
1 & \operatorname{Arg} z \in\left[0, \frac{1}{L} 2 \pi\right) \\
\vdots & \\
\omega_{L}^{l} & \operatorname{Arg} z \in\left[\frac{l}{L} 2 \pi, \frac{l+1}{L} 2 \pi\right), \\
\vdots & \\
\omega_{L}^{L-1} & \operatorname{Arg} z \in\left[\frac{L-1}{L} 2 \pi, 2 \pi\right)
\end{array},\right.
$$

where $z \in \mathbb{C}, \omega_{L}=\exp (2 \pi j / L)$ and $\operatorname{Arg} z$ is the angle of $z$. With the function $f_{L}$, the random vector $\xi$ generated from the complex Gaussian distribution $C \mathcal{N}\left(\mathbf{0}, X_{L}^{\star}\right)$ is transformed to

$$
\hat{b}_{p}=f_{L}\left(\xi_{p}\right) \quad(p=1,2, \ldots, P) .
$$

It is clear that $\hat{b}_{p} \in \Omega_{L}$. Therefore, $\hat{\mathbf{b}}$ is a feasible solution of the problem $\left(\hat{Q}_{L}\right)$. With the above method, we can obtain the feasible solutions from random vectors generated from the complex Gaussian distribution $\mathcal{C N}\left(\mathbf{0}, X_{L}^{\star}\right)$. Then, we choose the approximate solution from them which achieves the minimum PAPR among the set of the random vectors. Our method is summarized in Algorithm 1.

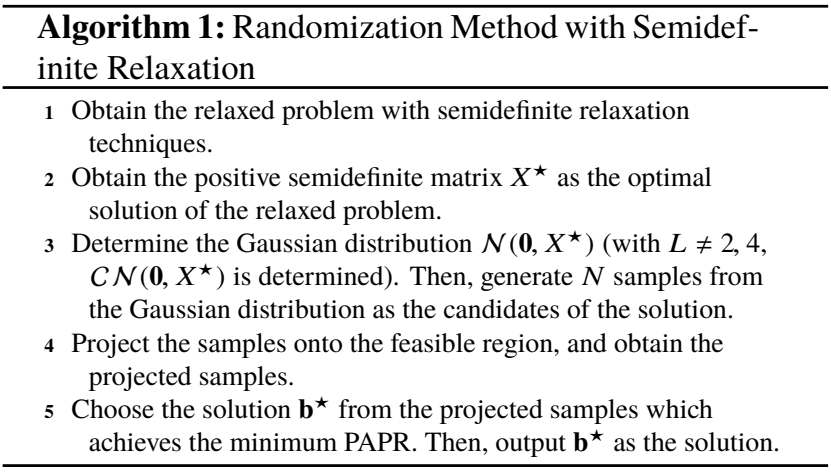

\subsection{Computational Complexity}

There are many discussions about computational complexities of PAPR reduction techniques. In this section, we discuss the computational complexity of our randomization method under the condition that the disjoint index subsets $\Lambda_{p}$ are given. The remaining complexities consists of three parts: (i) to generate random vectors, (ii) to choose the optimal vector from the random vectors, and (iii) to obtain the solution of the optimization problem.

In generating random vectors, computational complexity is in proportion to the number of random vectors. The computational complexity turns out to be $N \cdot c_{\text {gen }}$, where $N$ and $c_{\text {gen }}$ are the number of random vectors and the computational cost for generating each random vector, respectively. Similarly, in choosing the optimal vector from given random vectors, its computational complexity is $N \cdot J K \cdot c_{\text {cal }}$, where $c_{\text {cal }}$ is the computational cost for calculating the amplitude of each samples, $J$ and $K$ have been defined in Sects. 3 and 2 , respectively.

When we solve the optimization problem to obtain the solution, the computational complexity for solving the optimization problem is not clear since our optimization problem is not an ordinary semidefinite relaxation problem written in [39]. In this paper, we assume that the computational complexity of our optimization problem is nearly equivalent to one of ordinary semidefinite relaxation problems. Then, the worst case complexity is $O\left(\max \{m, n\}^{4} n^{1 / 2} \log (1 / \epsilon)\right)$, where $O$ represents the order, $m$ is the number of constraints, $n$ is the dimension of the matrix, and $\epsilon>0$ is solution accuracy [39]. For the general $L$, since the optimization problem $\left(\hat{Q}_{L}^{\prime}\right)$ has $(J K+P)$ constraints, the worst case complexity is $O\left((J K+P)^{4} P^{1 / 2} \log (1 / \epsilon)\right)$.

\section{Relation between Our Method and Phase Random Method}

In Sect. 5, we have shown our randomization method. Similar to our method, the phase random method has been proposed [28]. This method uses random vectors whose phase is uniformly distributed in $\Omega_{L}$. In this section, we discuss the relation between our method and the phase random method.

First, we explain the phase random method. We define the probability mass function as

$$
\operatorname{Pr}\left\{z=\omega_{L}^{l}\right\}=\frac{1}{L} \quad(l=0,1, \ldots, L-1),
$$

where $\omega_{L}=\exp (2 \pi j / L)$, which has been defined in Sect. 5 . Thus, $\omega_{L} \in \Omega_{L}$ and phases are uniformly distributed in $\Omega_{L}$. With this probability, the phase random method is summarized in Algorithm 2.

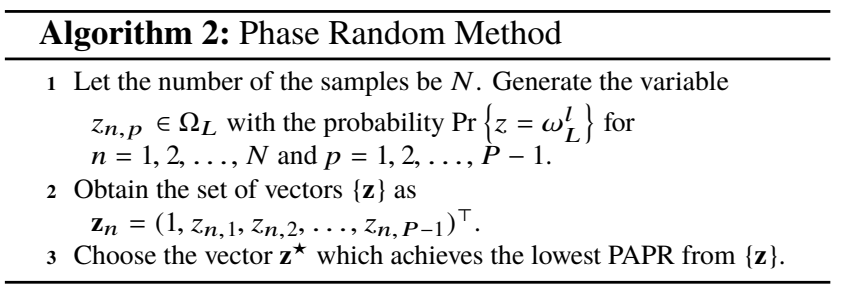


Further, let us discuss the complex Gaussian distribution. From [46], if $\mathbf{z} \in \mathbb{C}^{n}$ follows $C \mathcal{N}(\mu, \Sigma)$, then the probability density function of $\mathcal{T}(\mathbf{z}) \in \mathbb{R}^{2 n}$ is the Gaussian distribution $\mathcal{N}\left(\mathcal{T}(\boldsymbol{\mu}), \frac{1}{2} \mathcal{T}(\Sigma)\right)$. Therefore, we can consider a real-value Gaussian distribution instead of a complex Gaussian distribution.

Let us consider the complex Gaussian distribution $C \mathcal{N}\left(\mathbf{0}, I_{P}\right)$, where $I_{P}$ is the identity matrix whose size is $P$. It is clear that the matrix $\mathcal{T}\left(I_{P}\right)$ is the identity matrix whose size is $2 P$. From the above discussion, and the covariance matrix is identity matrix, each variable of $\mathbf{z}$ generated from $\mathcal{C N}\left(\mathbf{0}, I_{P}\right)$ is uncorrelated. It is known in [50] that uncorrelatedness is equivalent to independence for normal variables. Therefore, it is sufficient to consider a vector $\mathbf{z}$ whose element is generated from the complex Gaussian distribution $\mathcal{C N}(0,1)$. The variable $z$ which is the element of $\mathbf{z}$ can be decomposed as $z=x+j y$, where $x$ and $y$ are real numbers following the independent Gaussian distribution $\mathcal{N}(0,1 / 2)$, respectively.

Let us define $r \geq 0$ and $\theta \in[0,2 \pi)$ so that $x+j y=$ $r \exp (j \theta)$. Then, since $x$ and $y$ are normal variables following $\mathcal{N}(0,1 / 2)$, the probability density of $\theta \in[0,2 \pi)$ is

$$
p(\theta)=\frac{1}{2 \pi},
$$

from which, the phase of a variable $z$ generated from $C \mathcal{N}(0,1)$ is uniformly distributed [50].

From the above discussions and the definition of the function $f_{L}(z)$, the probability mass function of $f_{L}(z)$ is written as

$$
\operatorname{Pr}\left\{f_{L}(z)=\omega_{L}^{l}\right\}=\frac{1}{L} .
$$

This result implies that the phase random method is equivalent to our method whose covariance matrix is the identity matrix with the function $f_{L}(z)$.

\section{Reducing Upper Bound of PAPR}

We have discussed how to obtain a covariance matrix to determine a Gaussian distribution. In Sect. 5, we have obtained the optimization problem $\left(\hat{Q}_{L}^{\prime}\right)$. This problem contains the oversampling parameter $J$. As seen in Sect. 3, measured PAPR calculated from sampled signals converges to the true value of PAPR as $J \rightarrow \infty$. Therefore, a sufficiently large $J$ is necessary to evaluate PAPR tightly. Then, however, as seen in Sect. 5, the number of constraints in the optimization problem $\left(\hat{Q}_{L}^{\prime}\right)$ gets larger as $J$ increases. In such a situation, the optimization problem $\left(\hat{Q}_{L}^{\prime}\right)$ gets complicated.

To overcome this obstacle, instead of PAPR, we consider an optimization problem to reduce the upper bound of PAPR which does not depend on time $t$. From this problem, we obtain a covariance matrix as the solution.

In this section, we consider a general $L$. Then, specifying $L=2,4$, we can verify the same results for ones obtained in this section with the techniques discussed in Sect. 4 as follows. With $L=2$, the set of matrices is the symmetric matrices $\mathbb{S}_{P}$, and with $L=4$, we replace a positive semidefinite matrix $X$ with $\mathcal{T}(X)$.

The upper bound of the signal envelope has been shown with Eq. (1) as [51]

$$
|s(t)|^{2} \leq \sum_{k=1}^{K}\left|A_{k}\right|^{2}+2 \sum_{i=1}^{K-1}|\rho(i)|,
$$

where

$$
\rho(i)=\sum_{k=1}^{K-i} A_{k} \bar{A}_{k+i}
$$

and $\bar{z}$ is the complex conjugate of $z$. We let $\rho(K)=0$ for latter convenience. The right hand side of Eq. (39) is independent of the time $t$. Let us define $\rho^{\prime}=(\rho(1), \rho(2), \ldots, \rho(K-$ $1))^{\top}$. Note that the first term in right side of Eq. (39), $\sum_{k=1}^{K}\left|A_{k}\right|^{2}$, corresponds to $\rho(0)$ and this term is not varied with PTS techniques since each element of a vector $b_{n}$ satisfies $\left|b_{n}\right|=1$.

From the above discussion, without taking into account convexity, it is expected to decrease PAPR when we reduce $\left\|\boldsymbol{\rho}^{\prime}\right\|_{l_{1}}$, where $\|\mathbf{z}\|_{l_{1}}$ is the $l_{1}$-norm of $\mathbf{z}$. However, it is not the case since each $|\rho(i)|$ is not convex if we regard $A_{k}$ as variables. Therefore, we use $l_{2}$-norm of $\rho^{\prime}$, $\left\|\rho^{\prime}\right\|_{l_{2}}$. From the Cauchy-Schwarz inequality, it follows that $\left\|\rho^{\prime}\right\|_{l_{1}} \leq \sqrt{K-1}\left\|\rho^{\prime}\right\|_{l_{2}}$. Therefore, $\left\|\rho^{\prime}\right\|_{l_{1}}$ is expected to decrease when $\left\|\rho^{\prime}\right\|_{l_{2}}$ decreases.

Let us consider the following vector

$$
\hat{\boldsymbol{\rho}}=(\rho(0), \sqrt{2} \rho(1), \ldots, \sqrt{2} \rho(K-1))^{\top} .
$$

It is clear that minimizing $\|\hat{\rho}\|_{l_{2}}$ is equivalent to minimizing $\left\|\sqrt{2} \rho^{\prime}\right\|_{l_{2}}$ since $\rho(0)$ is constant. Then, $\|\hat{\rho}\|_{l_{2}}^{2}$ is written as

$$
\begin{aligned}
\|\hat{\boldsymbol{\rho}}\|_{l_{2}}^{2} & =2 \sum_{k=1}^{K-1}|\rho(k)|^{2}+|\rho(0)|^{2} \\
& =\sum_{k=0}^{K-1}|\rho(k)|^{2}+\sum_{k=0}^{K-1}|\rho(K-k)|^{2} \\
& =\frac{1}{2}\left\{\sum_{k=0}^{K-1}|\rho(k)+\overline{\rho(K-k))}|^{2}+|\rho(k)-\overline{\rho(K-k)}|^{2}\right\} .
\end{aligned}
$$

Here, $\overline{\rho(k)}$ denotes the complex conjugate of $\rho(k)$. From the above equations, $\|\hat{\rho}\|_{l_{2}}^{2}$ is divided into a periodic correlation term and an odd periodic correlation term. With Eq. (8), these terms are written as

$$
\begin{aligned}
& \rho(k)+\overline{\rho(K-k)}=\mathbf{b}^{*} A^{*} B_{1,1}^{(k)} A \mathbf{b}, \\
& \rho(k)-\overline{\rho(K-k)}=\mathbf{b}^{*} A^{*} B_{-1,1}^{(k)} A \mathbf{b},
\end{aligned}
$$

where the matrices $B_{1,1}^{(k)}$ and $B_{-1,1}^{(k)}$ are written as

$$
B_{1,1}^{(k)}=\left(\begin{array}{cc}
O & I_{k} \\
I_{K-k} & O
\end{array}\right), \quad B_{-1,1}^{(k)}=\left(\begin{array}{cc}
O & -I_{k} \\
I_{K-k} & O
\end{array}\right) .
$$


Since these matrices are regular, they can be transformed to diagonal matrices. With this general discussion, these matrices are decomposed as [52]

$$
B_{1,1}^{(k)}=V^{*} D^{(k)} V, B_{-1,1}^{(k)}=\hat{V}^{*} \hat{D}^{(k)} \hat{V}
$$

where $V$ and $\hat{V}$ are unitary matrices whose $(m, n)$-th elements are

$$
\begin{aligned}
& V_{m, n}=\frac{1}{\sqrt{K}} \exp \left(-2 \pi j \frac{m n}{K}\right), \\
& \hat{V}_{m, n}=\frac{1}{\sqrt{K}} \exp \left(-2 \pi j n\left(\frac{m}{K}+\frac{1}{2 K}\right)\right),
\end{aligned}
$$

and $D^{(k)}$ and $\hat{D}^{(k)}$ are diagonal matrices whose $n$-th diagonal elements are

$D_{n}^{(k)}=\exp \left(-2 \pi j k \frac{n}{K}\right), \hat{D}_{n}^{(k)}=\exp \left(-2 \pi j k\left(\frac{n}{K}+\frac{1}{2 K}\right)\right)$.

With these expressions, Eq. (42) is written as

$$
\|\hat{\boldsymbol{\rho}}\|_{l_{2}}^{2}=\frac{K}{2}\left\{\sum_{k=1}^{K}\left|\alpha_{k}\right|^{4}+\sum_{k=1}^{K}\left|\beta_{k}\right|^{4}\right\},
$$

where $\alpha_{k}$ and $\beta_{k}$ are the $k$-th element of $\boldsymbol{\alpha}$ and $\boldsymbol{\beta}$ written as $\boldsymbol{\alpha}=V A \mathbf{b}$ and $\boldsymbol{\beta}=\hat{V} A \mathbf{b}$, respectively. With the variable $\mathbf{b}$, the above equation is written as

$$
\|\hat{\boldsymbol{\rho}}\|_{l_{2}}^{2}=\frac{K}{2} \sum_{k=1}^{K}\left\{\left(\mathbf{b}^{*} A^{*} V^{*} G_{k} V A \mathbf{b}\right)^{2}+\left(\mathbf{b}^{*} A^{*} \hat{V}^{*} G_{k} \hat{V} A \mathbf{b}\right)^{2}\right\},
$$

where $G_{k}$ is a matrix whose $(k, k)$-th element is unity and the other elements are zero. Note that $G_{k}=G_{k}^{*} G_{k}$. Then, the matrices $A^{*} V^{*} G_{k} V A$ and $A^{*} \hat{V}^{*} G_{k} \hat{V} A$ are positive semidefinite matrices since they are the Gram matrices. Further, Eq. (49) is convex with respect to the variable b. This is proven in Appendix A. From the above discussions, it follows that the squared $l_{2}$-norm of $\hat{\rho}$ is a convex function with respect to the variable b. Combining these discussions above, we obtain the optimization problem,

$$
\begin{array}{cc}
\left(Q_{l_{2}}\right) & \min F(\mathbf{b}) \\
\text { subject to } & b_{p} \in \Omega_{L} \quad(p=1,2, \ldots, P),
\end{array}
$$

where

$$
F(\mathbf{b})=\sum_{k=1}^{K}\left\{\left(\mathbf{b}^{*} A^{*} V^{*} G_{k} V A \mathbf{b}\right)^{2}+\left(\mathbf{b}^{*} A^{*} \hat{V}^{*} G_{k} \hat{V} A \mathbf{b}\right)^{2}\right\} .
$$

To overcome the obstacle caused by the discreteness of $\Omega_{L}$, we obtain a relaxed problem with semidefinite relaxation techniques. This convex problem is written as

$$
\begin{aligned}
\left(Q_{l_{2}}^{\prime}\right) \quad & \min \hat{F}(X) \\
\text { subject to } & X_{p, p}=1 \quad(p=1,2, \ldots, P) \\
& X \geqslant 0, \quad X \in \mathbb{H}_{P},
\end{aligned}
$$

where

$$
\hat{F}(\mathbf{X})=\sum_{k=1}^{K}\left\{\left(A^{*} V^{*} G_{k} V A X\right)^{2}+\left(A^{*} \hat{V}^{*} G_{k} \hat{V} A X\right)^{2}\right\} .
$$

From the above discussions, how to obtain the optimal solution as a positive semidefinite matrix has been shown. Then, we discuss the relations between our randomization method and the relaxed problem $\left(Q_{l_{2}}^{\prime}\right)$. Let $X^{\star}$ and $\{\xi\}$ be the global solution of the problem $\left(Q_{l_{2}}^{\prime}\right)$ and the random vectors generated from the Gaussian distribution $C \mathcal{N}\left(\mathbf{0}, X^{\star}\right)$, respectively. They satisfy $\mathrm{E}\left\{\boldsymbol{\xi} \xi^{*}\right\}=X^{\star}$. Then, it follows that

$$
\begin{aligned}
& \sum_{k=1}^{K}\left\{\operatorname{Tr}\left(A^{*} V^{*} G_{k} V A X^{\star}\right)^{2}+\operatorname{Tr}\left(A^{*} \hat{V}^{*} G_{k} \hat{V} A X^{\star}\right)^{2}\right\} \\
\leq & \sum_{k=1}^{K} \mathrm{E}\left\{\left(\xi^{*} A^{*} V^{*} G_{k} V A \xi\right)^{2}+\left(\xi^{*} A^{*} \hat{V}^{*} G_{k} \hat{V} A \xi\right)^{2}\right\} \\
\leq & 3 \sum_{k=1}^{K}\left\{\operatorname{Tr}\left(A^{*} V^{*} G_{k} V A X^{\star}\right)^{2}+\operatorname{Tr}\left(A^{*} \hat{V}^{*} G_{k} \hat{V} A X^{\star}\right)^{2}\right\} .
\end{aligned}
$$

The above relations are proven in Appendix B. Our main aim is to find $X_{l_{2}}^{\star}$ minimizing $\mathrm{E}\{F(\boldsymbol{\xi})\}$ under the constraints, where $\boldsymbol{\xi} \sim \mathcal{C N}\left(\mathbf{0}, X_{l_{2}}^{\star}\right)$. Two inequalities are involved in Eq. (54). The first inequality in Eq. (54) implies that the global solution of the relaxed problem $X^{\star}$ does not always correspond to $X_{l_{2}}^{\star}$. However, the last inequality in Eq. (54) implies that $X^{\star}$ will be an appropriate solution for our randomization method since $X^{\star}$ will make $\mathrm{E}\{F(\boldsymbol{\xi})\}$ small where $\xi \sim C \mathcal{N}\left(\mathbf{0}, X^{\star}\right)$. From the above discussions, the global solution of the relaxed problem $X^{\star}$ is not the optimal covariance matrix with our randomization method minimizing upper bound of PAPR. However, $X^{\star}$ will achieve low PAPR with our randomization method.

\section{Numerical Results}

In this section, from the perspective of PAPR and Bit Error Rate (BER), we compare the performances of our randomization methods with ones of the phase random method and the neighborhood method [27].

Here, we randomly generate transmitted symbols $\left\{A_{k}\right\}$. In practical situations, an error correction code is used and transmitted symbols depend on the code. However, it is known that most good codes do not introduce correlation into the symbol stream [8]. This yields that the transmitted symbols are also uncorrelated. Further, as mentioned in [8], a symbol stream is only locally dependent for traditional 
codes. Thus, the following numerical results shown in this section will be extended to the case where an error correction code is used.

We numerically solve the problems $\left(\hat{Q}_{L}^{\prime}\right)$ and $\left(Q_{l_{2}}^{\prime}\right)$ with CVX [53] and obtain approximate solutions with the two kinds of methods, the $l_{2}$ approximation method discussed in Sect. 4 and a random method discussed in Sects. 5 and 7 , respectively. As the parameters, the number of carriers $K=256$ and the oversampling parameter $J=16$ are chosen. We obtain PAPR curves with three kinds of parameters, $(P, L)=(16,2),(8,4)$ and $(8,8)$. The oversampling parameter $J$ is also used in calculating PAPR (see Eq. (15)). As the modulation scheme, each symbol is independently chosen from 16-QAM symbols. The index sets $\Lambda_{n}$ are randomly chosen from the set $\{1,2, \ldots, K\}$ and they satisfy $\left|\Lambda_{n}\right|=\frac{K}{P}$ for $n=1,2, \ldots P$, where $\left|\Lambda_{n}\right|$ is the number of components in the set $\Lambda_{n}$. It has been known that PAPR with random index sets is lower than one with adjacent index sets [29]. With our randomization methods discussed in Sects. 5 and 7, and the phase random method, we generate 10 and 70 samples as solution candidates and choose the optimal solution from such candidates (see Algorithm 1). For the brute force method and the other methods, we draw the PAPR curves from 200 results and 2000 results, respectively. Note that the PAPR curve with the brute force method is optimal. For the neighborhood method, we set the Hamming weight parameter $r=1$ and the maximum iteration parameter $I=5,4$ and 3 in $(P, L)=(16,2),(8,4)$ and $(8,8)$, respectively. Note that the number of candidates in the neighborhood method is roughly calculated as $I(P-1)(L-1)$ when $r=1$. With these parameters, the number of candidates in the neighborhood method is roughly calculated as 70 . Thus, the search complexity of the neighborhood method is nearly equivalent to one of our methods.

Figures 1, 2 and 3 show each PAPR curve with original OFDM systems, the brute force method [23], the $l_{2}$ approximation method discussed in Sect. 4, the randomization method discussed in Sect. 5, the reducing-upper bound method discussed in Sect. 7, the phase random method [28] and the neighborhood method [27]. In the legends, " $l_{2}$ approximation", "Ours (PAPR)", "Ours (Upper Bound)" and "Neighborhood" mean the $l_{2}$ approximation method, the randomization method discussed in Sect. 5, the reducing-upper bound method discussed in Sect. 7 and the neighborhood method, respectively. In Fig. 3, the PAPR curve with the brute force method is not drawn since it is not straightforward to obtain the optimal vector due to its significantly large calculation amount. From these figures, the PAPR curve with the $l_{2}$ approximation method is far from one with the brute force method. This result shows that the optimal solution of the relaxed problem is far from a rank-1 matrix and it tends to have some large eigenvalues. Therefore, we conclude that the $l_{2}$ approximation method is not suitable for PTS techniques.

With randomization methods, there are two PAPR curves obtained from 10 random vectors and 70 random vectors. In both numbers of random vectors, the PAPRs

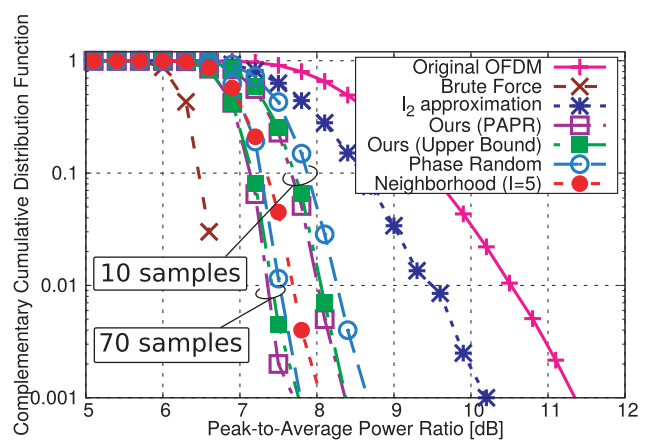

Fig. 1 PAPR with the 16-QAM modulation scheme in the parameters $(P, L)=(16,2), K=256$ and $J=16$.

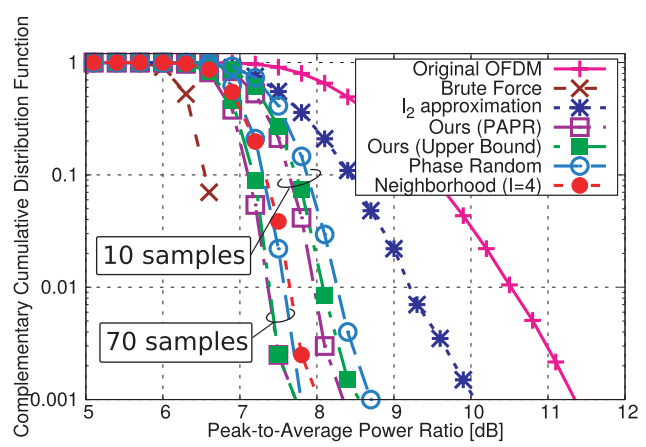

Fig. 2 PAPR with the 16-QAM modulation scheme in the parameters $(P, L)=(8,4), K=256$ and $J=16$.

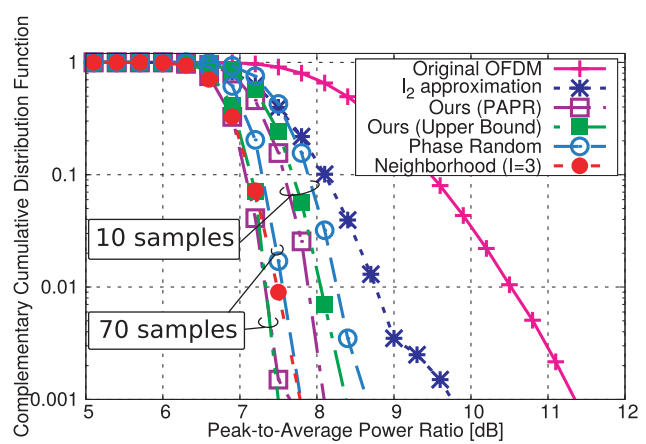

Fig. 3 PAPR with the 16-QAM modulation scheme in the parameters $(P, L)=(8,8), K=256$ and $J=16$

of our two randomization methods are lower than those of phase random techniques. As seen in Sect. 6, the phase random method is equivalent to our method with the identical matrix as a covariance matrix. Therefore, the performance of randomization methods can be improved when a suitable covariance matrix is chosen. Further, with 70 samples, PAPRs of our two randomization methods are lower than one of the neighborhood method. Since the search region of the neighborhood method is roughly equivalent to ones of our methods, these results show that our methods can find a suitable vector more efficiently than the neighborhood method.

In Sect. 5, we have discussed the computational complexity of our method. Tables 1, 2 and 3 show the averaged calculation time for obtaining a solution in our meth- 
Table 1 Averaged calculation time for algorithms with the parameters $(P, L)=(16,2), J=16$ and the 16-QAM modulation scheme.

\begin{tabular}{|c||c|c|c|}
\hline & Ours(PAPR) & Ours(Upper Bound) & Phase Random \\
\hline 10 iterations & $30.79111[\mathrm{sec}]$ & $5.442301[\mathrm{sec}]$ & $0.3556757[\mathrm{sec}]$ \\
\hline 70 iterations & $33.80662[\mathrm{sec}]$ & $6.900336[\mathrm{sec}]$ & $2.458494[\mathrm{sec}]$ \\
\hline
\end{tabular}

Table 2 Averaged calculation time for algorithms with the parameters $(P, L)=(8,4), J=16$ and the 16-QAM modulation scheme.

\begin{tabular}{|c||c|c|c|}
\hline & Ours(PAPR) & Ours(Upper Bound) & Phase Random \\
\hline 10 iterations & $32.78214[\mathrm{sec}]$ & $5.167035[\mathrm{sec}]$ & $0.291365[\mathrm{sec}]$ \\
\hline 70 iterations & $32.86061[\mathrm{sec}]$ & $6.119004[\mathrm{sec}]$ & $2.04702[\mathrm{sec}]$ \\
\hline
\end{tabular}

Table 3 Averaged calculation time for algorithms with the parameters $(P, L)=(8,8), J=16$ and the 16-QAM modulation scheme.

\begin{tabular}{|c||c|c|c|}
\hline & Ours(PAPR) & Ours(Upper Bound) & Phase Random \\
\hline 10 iterations & $20.40021[\mathrm{sec}]$ & $4.669453[\mathrm{sec}]$ & $0.2870807[\mathrm{sec}]$ \\
\hline 70 iterations & $22.47077[\mathrm{sec}]$ & $6.416054[\mathrm{sec}]$ & $2.060964[\mathrm{sec}]$ \\
\hline
\end{tabular}

Table 4 Averaged calculation time for the algorithms in the parameters $(P, L)=(16,2)$ and the 16-QAM modulation scheme.

\begin{tabular}{|c||c|c|c|}
\hline & $\boldsymbol{J}=4$ & $\boldsymbol{J}=8$ & $\boldsymbol{J}=16$ \\
\hline 10 iterations & $6.513275[\mathrm{sec}]$ & $13.62168[\mathrm{sec}]$ & $30.79111[\mathrm{sec}]$ \\
\hline 70 iterations & $8.060944[\mathrm{sec}]$ & $15.30768[\mathrm{sec}]$ & $33.80662[\mathrm{sec}]$ \\
\hline
\end{tabular}

ods and the phase random method with the parameters $(P, L)=(16,2),(8,4)$ and $(8,8)$, respectively. In these parameters, the averaged calculation time of the algorithm proposed in Sect. 5 and the reducing-upper bound method is larger than one of the phase random method. Since an optimization has to be solved to obtain a solution in our proposed method, the calculation time of our methods is larger than one of the phase random method. In our methods, the calculation time of the reducing-upper bound method is smaller than one of the method proposed in Sect. 5. The reason may be explained as follows. As seen in Sect. 5, it is expected that the computational complexity depends on the number of constraints which is written in terms of $J$. In the method proposed in Sect. 5 , the over sampling parameter $J=16$ is chosen here. Thus, the number of constraints of the method proposed in Sect. 5 is $J K+P=16 K+P$. On the other hand, the number of constraints in the reducing-upper bound method is regarded as $2 K$ since the objective function of this method consists of $2 K$ terms. Thus, the calculation time of the reducing-upper bound method is smaller than one of the method proposed in Sect. 5 since the number of the constraints in the reducing-upper bound method is smaller than one of the method proposed in Sect. 5. Thus, these results imply that the large number of constraints implies the large calculation complexity.

We have seen the calculation time for obtaining a solution with $J=16$. From the above discussion, it is expected that the calculation time gets smaller as the parameter $J$ gets smaller since the computational complexity will depend on $J$. Table 4 shows the averaged calculation time for obtaining a solution with several $J$ in the parameters $(P, L)=(16,2)$. Here, when we choose the solution from the candidate set, we use the parameter $J=16$ to calculate PAPR. Thus, only the parameter $J$ in the optimization problem is varied. As seen in Table 4, the averaged calculation time gets larger as $J$ gets larger. Note that the oversampling parameter $J$ in the reducing-upper bound method is regarded as $J=2$. This yields that the computational complexity gets larger as the parameter $J$ gets larger. Figure 4 shows PAPR curves with several $J$ for the parameters $(P, L)=(16,2)$. As seen in this figure, PAPRs of the methods proposed in Sect. 5 with $J=4,8$ and 16 are lower than one of the phase random method. From the above discussions, we conclude that the method proposed in Sect. 5 will be efficient with any $J$. Note that the parameter $J$ satisfies the condition defined in Eq. (15).

In the method proposed in Sect. 5, we have considered the three cases: (i) $L=2$, (ii) $L=4$, and (iii) general $L$. It is clear that the method for general $L$ can be applied when $L=2$ or $L=4$. Here, we verify the efficiency of our method for a general $L$ in such cases. Figures 5 and 6 show PAPR curves with the algorithm for a general $L$. In the legend, "PAPR" and "Upper Bound" denote the method proposed in Sect. 5 and the reducing-upper bound method, respectively. To draw these two figures, the parameters are chosen as $(P, L)=(16,2)$ and $(P, L)=(8,4)$, respectively. Here, the number of carriers and the parameter $J$ are $K=256$ and $J=16$. These figures show that PAPR of the method proposed in Sect. 5 for a general $L$ is lower than one of the phase random method. Thus, even in cases $L=2$ and $L=4$, our method for a general $L$ is efficient.

We have seen PAPRs obtained with our methods and some existing methods. These results have been obtained with the 16-QAM modulation scheme and the number of 


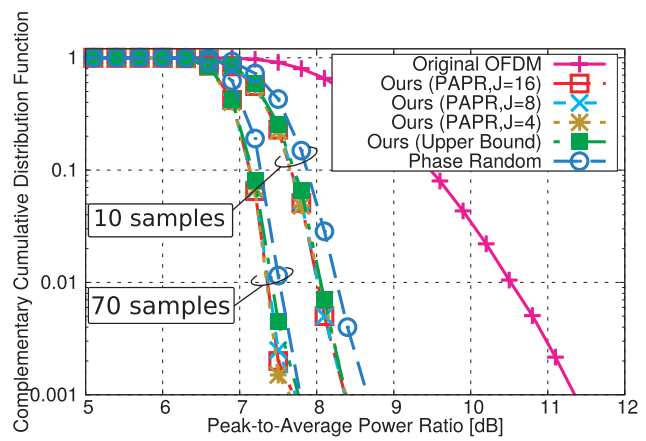

Fig. 4 PAPRs with some $J$ in the parameters $(P, L)=(16,2), K=256$ and the 16-QAM modulation scheme.

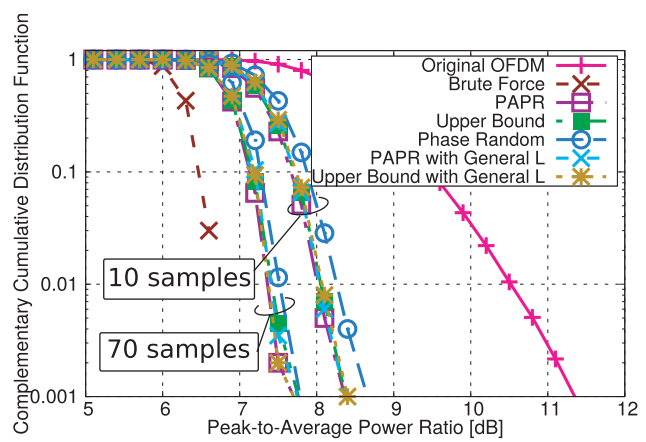

Fig.5 PAPR with the general $L$-randomization algorithm in the parameters $(P, L)=(16,2), K=256, J=16$ and the 16-QAM modulation scheme.

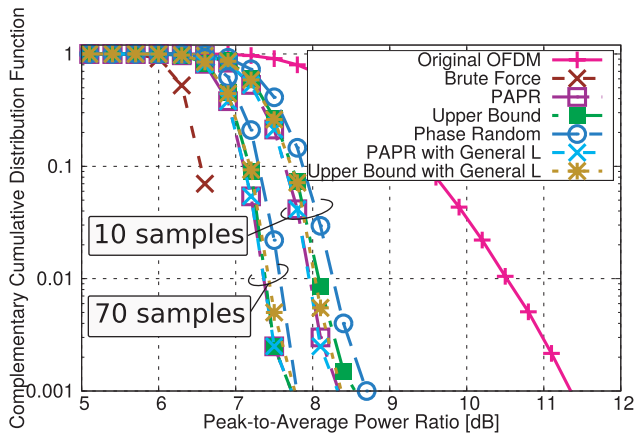

Fig. 6 PAPR with the general $L$-randomization algorithm in the parameters $(P, L)=(8,4), K=256, J=16$ and the 16-QAM modulation scheme.

carriers $K=256$. Here, we show some results with other situations. Figure 7 shows PAPR curves with $K=128$ for the parameters $(P, L)=(16,2)$. Here, the modulation scheme is the 16-QAM. Similar to the results in $K=256$, PAPRs of our methods are lower than one of the phase random method. Thus, our methods will be efficient for any $K$. Figures 8 and 9 show PAPR curves for the parameters $(P, L)=(16,2)$ and $K=256$ in the QPSK modulation scheme and 64-QAM modulation scheme, respectively. Similar to the results in the 16-QAM modulation scheme, our methods can achieve lower PAPRs than the phase random method in the both schemes. From the above discussions, we conclude that our

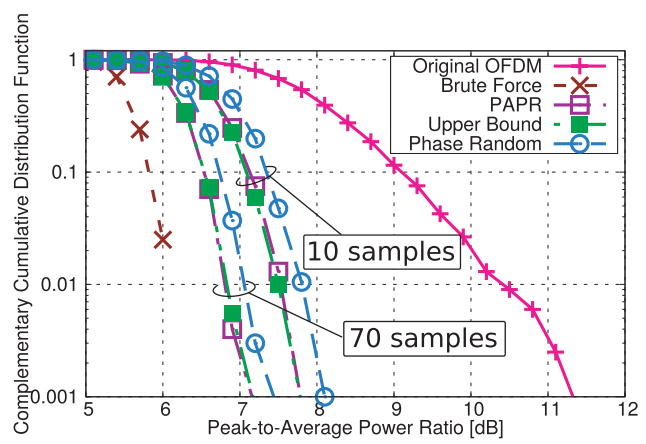

Fig. 7 PAPR with the 16-QAM modulation scheme in the parameters $(P, L)=(16,2), K=128$ and $J=16$.

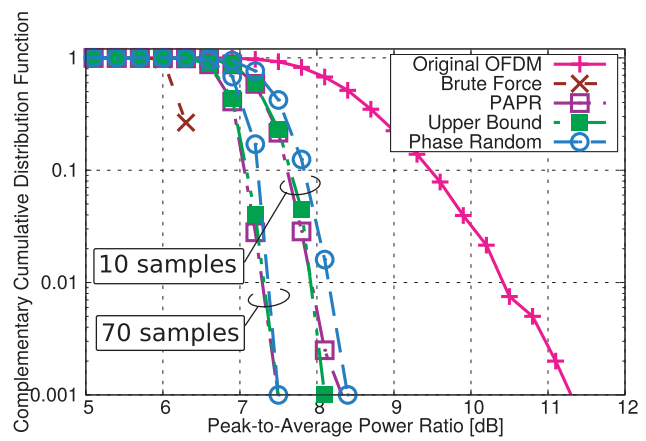

Fig. 8 PAPR with the QPSK modulation in the parameters $(P, L)=$ $(16,2), K=256$ and $J=16$.

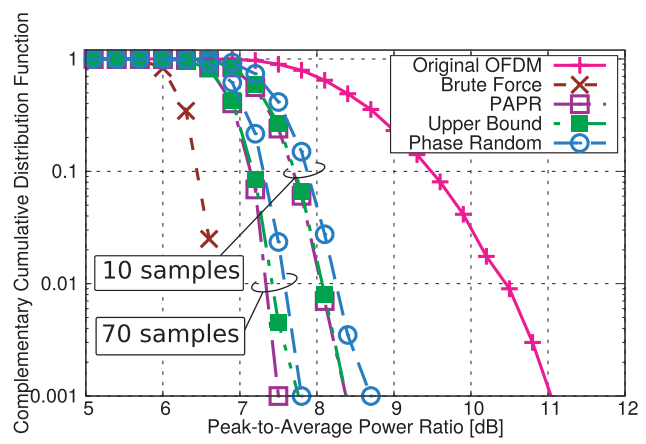

Fig. 9 PAPR with the 64-QAM modulation in the parameters $(P, L)=$ $(16,2), K=256$ and $J=16$.

method will be efficient with any number of carriers and any modulation scheme.

To explore the performances with our method and the phase random method, we evaluate each BER. To this end, the amplifier model is chosen as the Rapp model [54], which is described below. Let the input signal be presented in polar coordinates,

$$
x(t)=\rho(t) \exp (j \theta(t)) .
$$

Then, the output signal is written as

$$
\zeta(x(t))=\gamma(\rho(t)) \cdot \exp (j \cdot(\theta(t)+\Phi(\rho(t)))),
$$

where $\gamma$ and $\Phi$ are functions of the amplitude $\rho(t)$. In the 


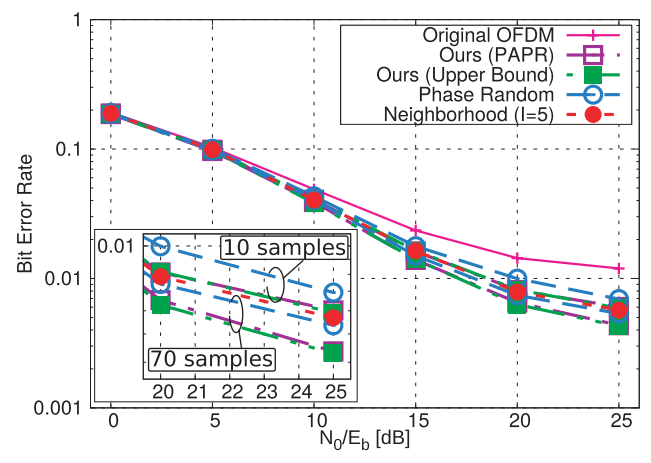

Fig. 10 BER with the 16-QAM modulation scheme in the parameters $(P, L)=(16,2), K=256$ and $J=16$.

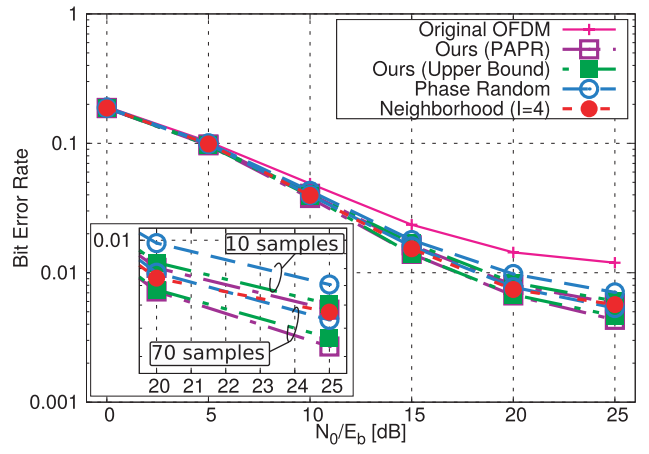

Fig. 11 BER with the 16-QAM modulation scheme in the parameters $(P, L)=(8,4), K=256$ and $J=16$.

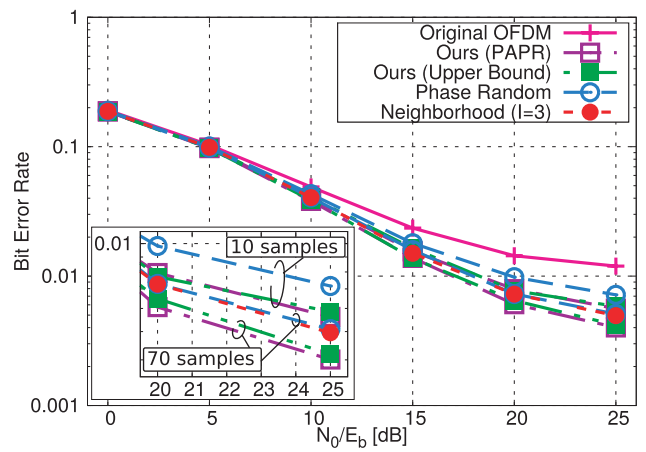

Fig. 12 BER with the 16-QAM modulation scheme in the parameters $(P, L)=(8,8), K=256$ and $J=16$.

Rapp model, these two functions are chosen as

$$
\gamma(\rho)=\frac{\rho}{\left(1+\left(\frac{\rho}{r}\right)^{p}\right)^{\frac{1}{2 p}}}, \quad \Phi(\rho)=0,
$$

where $\rho$ is an amplitude, $r$ is the clipping level and $p$ is the real parameter. The parameter $p$ is often chosen as $p=2$ or $p=3[55]-[57]$.

Figures 10, 11 and 12 show each BER curve with the parameter $(P, L)=(16,2),(8,4)$ and $(8,8)$. Here, the number of carriers $K=256$, the oversampling parameter $J=16$ and the modulation scheme is the 16-QAM. Further, we assume that the receiver knows the solution vector $\mathbf{b}$ which is used to modulate symbols and sent from the sender. Thus, we do not take into account the channel for side-information. In these figures, $E_{b}$ denotes the average energy per bit at the receiver which is need for a reliable recovery of the information. In the Rapp model, we set the parameters $p=2$ and $r=\sqrt{P_{\mathrm{av}}} 10^{\frac{1}{10}}(r=2[\mathrm{~dB}])$. The simulation environment is the same to one in calculating PAPR. As seen in Figs. 1-3, we have found that our methods discussed in Sects. 5 and 7 have lower PAPR than ones of the phase random method and the neighborhood method. From Figs. 10-12, with nearly the same search complexity, BER of our methods discussed in Sects. 5 and 7 is lower than the two kinds of methods, the phase random method and the neighborhood method. From the above results, we conclude that our methods can achieve low PAPR more efficiently than the phase random method and the neighborhood method.

In our numerical result, we have used random numbers to generate transmitted symbols and verified the performance with these random numbers. Thus, we consider the case where each carrier is regarded as independent. However, in general, OFDM systems with a error correction code have dependency carriers, that is, each carrier cannot be regarded as independent. In fact, there are some error correction codes which achieve small PAPR and their carriers will not be independent [9], [30]. In such a case, PAPR results may not be similar to ones obtained here. However, as seen in [8], most "good" codes for random errors do not introduce correlation into the symbol stream. Thus, using such a code is equivalent to using random number to generate transmitted symbols. Thus, it is expected that low PAPR can be achieved with our methods even in a case where such a good code is used.

From the above two kinds of the results, our methods can achieve lower PAPR and BER than the phase random method, the neighborhood method and conventional OFDM signals.

\section{Conclusion}

In this paper, we have discussed how to obtain a suitable vector for partial transmit sequence techniques and have proposed two randomization methods with semidefinite relaxation techniques. Further, we have shown the relation between our methods and the phase random method. Then, in our numerical results, we have shown their PAPR curves and that our methods can achieve lower PAPR than the phase random method and the neighborhood method. Moreover, our numerical results have implied that randomization methods can achieve lower PAPR if a more suitable covariance matrix is obtained.

We have considered the case where the transmitted symbols are randomly generate. Its plausibility has been shown in Sect. 8. In wireless communication systems, pilot symbols are often inserted. It will be demanded to take into account these pilot symbols to reduce PAPR.

A remaining issue is to explore how to obtain a suitable covariance matrix for a randomization method rapidly. As seen in the discussions in Sect. 5, it takes time to solve the 
optimization problem numerically to obtain the covariance matrix. Therefore, one of necessities to address computational complexity is to obtain the explicit form of a suitable covariance matrix. After giving such an explicit way, we expect an ideal method for obtaining low PAPR.

\section{Acknowledgment}

One of the authors (H. T) would like to thank Dr. Shin-itiro Goto for his advise. This work was partially supported by JSPS (KAKENHI) Grant Number 18J12903.

\section{References}

[1] H. Schulze and C. Lüders, Theory and Applications of OFDM and CDMA: Wideband Wireless Communications, John Wiley \& Sons, 2005

[2] B. Muquet, Z. Wang G.B. Giannakis, M. de Courville, and P. Duhamel, "Cyclic prefixing or zero padding for wireless multicarrier transmissions?," IEEE Trans. Commun., vol.50, no.12, pp.2136$2148,2002$.

[3] E. Biglieri, R. Calderbank, A. Constantinides, A. Goldsmith, A. Paulraj, and H.V. Poor, MIMO Wireless Communications, Cambridge University Press, 2007.

[4] B. Farhang-Boroujeny, "OFDM versus filter bank multicarrier," IEEE Signal Process. Mag., vol.28, no.3, pp.92-112, 2011.

[5] H. Rohling, ed., OFDM: Concepts for Future Communication Systems, Springer Science \& Business Media, 2011

[6] H. Ochiai and H. Imai, "On the distribution of the peak-to-average power ratio in OFDM signals," IEEE Trans. Commun., vol.49, no.2, pp.282-289, 2001.

[7] T. Jiang, M. Guizani, H.H. Chen, W. Xiang, and Y. Wu, "Derivation of PAPR distribution for OFDM wireless systems based on extreme value theory,” IEEE Trans. Wireless Commun., vol.7, no.4, pp.12981305, 2008.

[8] S. Wei, D.L. Goeckel, and P.A. Kelly, "Convergence of the complex envelope of bandlimited OFDM signals," IEEE Trans. Inf. Theory, vol.56, no.10, pp.4893-4904, 2010.

[9] S. Litsyn and G. Wunder, "Generalized bounds on the crest-factor distribution of OFDM signals with applications to code design," IEEE Trans. Inf. Theory, vol.52, no.3, pp.992-1006, 2006.

[10] H. Ochiai and H. Imai, "Performance analysis of deliberately clipped OFDM signals," IEEE Trans. Commun., vol.50, no.1, pp.89-101, 2002.

[11] E. Costa and S. Pupolin, "M-QAM-OFDM system performance in the presence of a nonlinear amplifier and phase noise," IEEE Trans. Commun., vol.50, no.3, pp.462-472, 2002

[12] R.W. Bauml, R.F.H. Fischer, and J.B. Huber, "Reducing the peak-toaverage power ratio of multicarrier modulation by selected mapping," Electron. Lett., vol.32, no.22, pp.2056-2057, 1996.

[13] M. Sharif and B. Hassibi, "Existence of codes with constant PMEPR and related design," IEEE Trans. Signal Process., vol.52, no.10, pp.2836-2846, 2004

[14] B.S. Krongold and D.L. Jones, "PAR reduction in OFDM via active constellation extension," IEEE Trans. Broadcast., vol.49, no.3, pp.258-268, 2003.

[15] N. Jacklin and Z. Ding, "A linear programming based tone injection algorithm for PAPR reduction of OFDM and linearly precoded systems," IEEE Trans. Circuits \& Syst. I, Reg. Papers, vol.60, no.7, pp.1937-1945, 2013.

[16] T. Jiang and G. Zhu, "Complement block coding for reduction in peak-to-average power ratio of OFDM signals," IEEE Commun. Mag., vol.43, no.9, pp.S17-S22, 2005.

[17] C. Li, T. Jiang, Y. Zhou, and H. Li, "A novel constellation reshaping method for PAPR reduction of OFDM signals," IEEE Trans. Signal
Process., vol.59, no.6, pp.2710-2719, 2011.

[18] Y.-C. Wang and Z.-Q. Luo, "Optimized iterative clipping and filtering for PAPR reduction of OFDM signals," IEEE Trans. Commun., vol.59, no.1, pp.33-37, 2011

[19] Y. Jiang, "New companding transform for PAPR reduction in OFDM,” IEEE Commun. Lett., vol.14, no.4, pp.282-284, 2010.

[20] T. Jiang and Y. Wu, "An overview: Peak-to-average power ratio reduction techniques for OFDM signals," IEEE Trans. Broadcast., vol.54, no.2, pp.257-268, 2008.

[21] S. Litsyn, Peak Power Control in Multicarrier Communications, Cambridge University Press, 2007.

[22] F. Sandoval, G. Poitau, and F. Gagnon, "Hybrid peak-to-average power ratio reduction techniques: Review and performance comparison," IEEE Access, vol.5, pp.27145-27161, 2017.

[23] S.H. Muller and J.B. Huber, "OFDM with reduced peak-to-average power ratio by optimum combination of partial transmit sequences," Electron. Lett., vol.33, no.5, pp.368-369, 1997.

[24] K.S. Lee, H. Kang, and J.S. No, "New PTS schemes with adaptive selection methods of dominant time-domain samples in OFDM systems," IEEE Trans. Broadcast., vol.64, no.3, pp.747-761, 2018.

[25] H. Tsuda and K. Umeno, "Semidefinite relaxation based randomization approach for partial transmit sequence," IEEE Wireless Communications and Networking Conference (WCNC), 2018.

[26] H. Tsuda and K. Umeno, "Randomization algorithm for partial transmit sequence with semidefinite relaxation," IEEE Vehicular Technology Conference (VTC Fall), 2018.

[27] S.H. Han and J.H. Lee, "PAPR reduction of OFDM signals using a reduced complexity PTS technique," IEEE Signal Process. Lett., vol.11, no.11, pp.887-890, 2004

[28] L.J. Cimini and N.R. Sollenberger, "Peak-to-average power ratio reduction of an OFDM signal using partial transmit sequences," IEEE Commun. Lett., vol.4, no.3, pp.86-88, 2000.

[29] S.H. Muller and J.B. Huber, "A novel peak power reduction scheme for OFDM," Personal, Indoor and Mobile Radio Commun., Proc., The 8th IEEE International Symposium on., vol.3, IEEE, 1997.

[30] K.G. Paterson and V. Tarokh, "On the existence and construction of good codes with low peak-to-average power ratios," IEEE Trans. Inf. Theory, vol.46, no.6, pp.1974-1987, 2000.

[31] V. Tarokh and H. Jafarkhani, "On the computation and reduction of the peak-to-average power ratio in multicarrier communications," IEEE Trans. Commun., vol.48, no.1, pp.37-44, 2000.

[32] M. Sharif, M. Gharavi-Alkhansari, and B.H. Khalaj, "On the peakto-average power of OFDM signals based on oversampling," IEEE Trans. Commun., vol.51, no.1, pp.72-78, 2003.

[33] S.H. Muller and J.B. Huber, "A comparison of peak power reduction schemes for OFDM," Global Telecommunications Conference, 1997. GLOBECOM'97., IEEE., vol.1, IEEE, 1997.

[34] D.W. Lim, S.J. Heo, J.S. No, and H. Chung, "A new PTS OFDM scheme with low complexity for PAPR reduction," IEEE Trans. Broadcast., vol.52, no.1, pp.77-82, 2006.

[35] A. Ghassemi and T.A. Gulliver, "PAPR reduction of OFDM using PTS and error-correcting code subblocking-transactions papers," IEEE Trans. Wireless Commun., vol.9, no.3, pp.980-989, 2010.

[36] G. Wunder and H. Boche, "Peak value estimation of bandlimited signals from their samples, noise enhancement, and a local characterization in the neighborhood of an extremum," IEEE Trans. Signal Process., vol.51, no.3, pp.771-780, 2003.

[37] C. Tellambura, "Computation of the continuous-time PAR of an OFDM signal with BPSK subcarriers," IEEE Commun. Lett., vol.5, no.5, pp.185-187, 2001.

[38] W.K. Ma, B.N. Vo, T.N. Davidson, and P.C. Ching, "Blind ML detection of orthogonal space-time block codes: Efficient highperformance implementations," IEEE Trans. Signal Process., vol.54, no.2, pp.738-751, 2006.

[39] Z.Q. Luo, W.K. Ma, A.M.C. So, Y. Ye, and Sh. Zhang, "Semidefinite relaxation of quadratic optimization problems," IEEE Signal Process. Mag., vol.27, no.3, pp.20-34, 2010. 
[40] S. Boyd and L. Vandenberghe, Convex Optimization, Cambridge University Press, 2004

[41] I. Waldspurger, A. d'Aspremont, and S. Mallat, "Phase recovery, maxcut and complex semidefinite programming," Math. Program., vol.149, no.1-2, pp.47-81, 2015.

[42] M.X. Goemans and D. Williamson, "Approximation algorithms for MAX-3-CUT and other problems via complex semidefinite programming," Proc. Thirty-Third Annual ACM Symposium on Theory of Computing, ACM, 2001

[43] Z.Q. Luo, N.D. Sidiropoulos, P. Tseng, and S. Zhang, "Approximation bounds for quadratic optimization with homogeneous quadratic constraints," SIAM J. Optim., vol.18, no.1, pp.1-28, 2007.

[44] R.J. Muirhead, Aspects of Multivariate Statistical Theory, Wiley Series in Probability and Mathematical Statistics, Wiley, New York, 1982.

[45] Z.Q. Luo and T.H. Chang, "SDP relaxation of homogeneous quadratic optimization: Approximation bounds and applications," Convex Optimization in Signal Processing and Communications, pp.117-165, 2010

[46] H.H. Andersen, M. Højbjerre, D. Sørensen, and P.S. Eriksen, Linear and Graphical Models: For the Multivariate Complex Normal Distribution, LNS, vol.101, Springer Science \& Business Media, 1995.

[47] S. Zhang and Y. Huang, "Complex quadratic optimization and semidefinite programming," SIAM J. Optim., vol.16, no.3, pp.871890, 2006.

[48] A.M.C. So, J. Zhang, and Y. Ye, "On approximating complex quadratic optimization problems via semidefinite programming relaxations," Math. Program., vol.110, no.1, pp.93-110, 2007.

[49] A. De Maio, S. De Nicola, Y. Huang, Z.Q. Luo, and S. Zhang, "Design of phase codes for radar performance optimization with a similarity constraint," IEEE Trans. Signal Process., vol.57, no.2, pp.610-621, 2009.

[50] A. Papoulis and S.U. Pillai, Probability, Random Variables, and Stochastic Processes, Tata McGraw-Hill Education, 2002.

[51] C. Tellambura, "Upper bound on peak factor of N-multiple carriers," Electron. Lett., vol.33, no.19, pp.1608-1609, 1997.

[52] H. Tsuda and K. Umeno, "Non-linear programming: Maximize SINR for designing spreading sequence," IEEE Trans. Commun., vol.66, no.1, pp.278-289, 2018.

[53] M. Grant, S. Boyd, and Y. Ye, "CVX: Matlab software for disciplined convex programming," 2008.

[54] C. Rapp, "Effects of HPA-nonlinearity on a 4-DPSK/OFDM-signal for a digital sound broadcasting signal," ESA, Second European Conference on Satellite Communications (ECSC-2), pp.179-184 (SEE N92-15210 06-32), 1991

[55] S.A. Aburakhia, E.F. Badran, and D.A.E. Mohamed, "Linear companding transform for the reduction of peak-to-average power ratio of OFDM signals," IEEE Trans. Broadcast., vol.55, no.1, pp.155-160, 2009

[56] J. Hou, X. Zhao, F. Gong, F. Hui, and J. Ge, "PAPR and PICR reduction of OFDM signals with clipping noise-based tone injection scheme,” IEEE Trans. Veh. Technol., vol.66, no.1, pp.222-232, 2017.

[57] R. Van Nee and A. De Wild, "Reducing the peak-to-average power ratio of OFDM," Vehicular Technology Conference, 1998. VTC 98. 48th IEEE., vol.3, IEEE, 1998.

[58] R.T. Rockafellar, Convex Analysis, Princeton University Press, 1972.

[59] E. Telatar, "Capacity of multi-antenna Gaussian channels," Trans. Emerging Telecommun. Tech., vol.10, no.6, pp.585-595, 1999.

[60] T.W. Anderson, An Introduction to Multivariate Statistical Analysis, vol.2, Wiley, New York, 1958.

\section{Appendix A： Proof of Convexity of Eq. (49)}

In this appendix, we prove that the function defined in Eq. (49)

$$
\|\hat{\boldsymbol{\rho}}\|_{l_{2}}^{2}=\frac{K}{2} \sum_{k=1}^{K}\left\{\left(\mathbf{b}^{*} A^{*} V^{*} G_{k} V A \mathbf{b}\right)^{2}+\left(\mathbf{b}^{*} A^{*} \hat{V}^{*} G_{k} \hat{V} A \mathbf{b}\right)^{2}\right\},
$$

is convex with respect to $\mathbf{b}$.

First, it follows that the matrices $A^{*} V^{*} G_{k} V A$ and $A^{*} \hat{V}^{*} G_{k} \hat{V} A$ are positive semidefinite matrices since they are the Gram matrices. To prove the convexity of the above function, it is sufficient to prove that each term of the above function is convex since the sum of convex functions is convex. Therefore, we prove

$$
\begin{aligned}
& \gamma\left(\mathbf{b}_{1}^{*} G \mathbf{b}_{1}\right)^{2}+(1-\gamma)\left(\mathbf{b}_{2}^{*} G \mathbf{b}_{2}\right)^{2} \\
\geq & \left(\left(\gamma \mathbf{b}_{1}+\left(1-\gamma \mathbf{b}_{2}\right)^{*} G\left(\gamma \mathbf{b}_{1}+(1-\gamma) \mathbf{b}_{2}\right)\right)^{2},\right.
\end{aligned}
$$

where $\gamma \in[0,1], \mathbf{b}_{1}, \mathbf{b}_{2} \in \mathbb{C}^{P}$ and $G$ is a positive semidefinite matrix corresponding to either $A^{*} V^{*} G_{n} V A$ or $A^{*} \hat{V}^{*} G_{n} \hat{V} A$.

Let us prove the convexity. Since $x^{2}$ is a convex and non-decreasing function for $x \geq 0$ and $\mathbf{b}^{*} G \mathbf{b}$ is convex and non-negative, the following inequalities are satisfied

$$
\begin{aligned}
& \left(\left(\gamma \mathbf{b}_{1}+(1-\gamma) \mathbf{b}_{2}\right)^{*} G\left(\gamma \mathbf{b}_{1}+(1-\gamma) \mathbf{b}_{2}\right)\right)^{2} \\
\leq & \left(\gamma \mathbf{b}_{1}^{*} G \mathbf{b}_{1}+(1-\gamma) \mathbf{b}_{2}^{*} G \mathbf{b}_{2}\right)^{2} . \\
\leq & \gamma\left(\mathbf{b}_{1}^{*} G \mathbf{b}_{1}\right)^{2}+(1-\gamma)\left(\mathbf{b}_{2}^{*} G \mathbf{b}_{2}\right)^{2} .
\end{aligned}
$$

Applying the above inequalities to each term of Eq. (49), and the sum of convex functions is convex, we have that $\|\hat{\rho}\|_{l}^{2}$ in Eq. (49) is convex. Another proof has been obtained with the Theorem 5.1 written in [58].

\section{Appendix B: Proof of Relations in Eq. (54)}

In this appendix, we prove the following relations written in Eq. (54)

$$
\begin{aligned}
& \sum_{k=1}^{K}\left\{\operatorname{Tr}\left(A^{*} V^{*} G_{k} V A X^{\star}\right)^{2}+\operatorname{Tr}\left(A^{*} \hat{V}^{*} G_{k} \hat{V} A X^{\star}\right)^{2}\right\} \\
\leq & \sum_{k=1}^{K} \mathrm{E}\left\{\left(\xi^{*} A^{*} V^{*} G_{k} V A \xi\right)^{2}+\left(\xi^{*} A^{*} \hat{V}^{*} G_{k} \hat{V} A \xi\right)^{2}\right\} \\
\leq & 3 \sum_{k=1}^{K}\left\{\operatorname{Tr}\left(A^{*} V^{*} G_{k} V A X^{\star}\right)^{2}+\operatorname{Tr}\left(A^{*} \hat{V}^{*} G_{k} \hat{V} A X^{\star}\right)^{2}\right\} .
\end{aligned}
$$

for $\boldsymbol{\xi} \sim \mathcal{C N}\left(\mathbf{0}, X^{\star}\right)$.

A proof that the first inequality holds is given as follows. From the Cauchy-Schwarz inequality, it holds that

$$
\begin{aligned}
& \sum_{k=1}^{K}\left\{\operatorname{Tr}\left(A^{*} V^{*} G_{k} V A X^{\star}\right)^{2}+\operatorname{Tr}\left(A^{*} \hat{V}^{*} G_{k} \hat{V} A X^{\star}\right)^{2}\right\} \\
\leq & \sum_{k=1}^{K} \mathrm{E}\left\{\left(\xi^{*} A^{*} V^{*} G_{k} V A \xi\right)^{2}+\left(\xi^{*} A^{*} \hat{V}^{*} G_{k} \hat{V} A \xi\right)^{2}\right\} .
\end{aligned}
$$


Then, a proof that the last inequality holds is given as follows. Similar to Appendix A, it is sufficient to prove

$$
\mathrm{E}\left\{\left(\boldsymbol{\xi}^{*} G \boldsymbol{\xi}\right)^{2}\right\} \leq 3 \operatorname{Tr}\left(G X^{\star}\right)^{2}
$$

where $G$ is a Hermitian and positive semidefinite matrix and $\boldsymbol{\xi} \sim \mathcal{C N}\left(\mathbf{0}, X^{\star}\right)$. In [46], it has been shown that $\mathcal{T}(\mathbf{z}) \sim \mathcal{N}\left(\mathcal{T}(\boldsymbol{\mu}), \frac{1}{2} \mathcal{T}(\Sigma)\right)$ if $\mathbf{z} \sim \mathcal{C N}(\mu, \Sigma)$. Note that the matrices $\mathcal{T}\left(X^{\star}\right)$ and $\mathcal{T}(G)$ are symmetric and positive semidefinite since $G$ and $X^{\star}$ are Hermitian and positive semidefinite [59]. From this result, it follows that $\mathcal{T}(\boldsymbol{\xi}) \sim \mathcal{N}\left(\mathcal{T}(\mathbf{0}), \frac{1}{2} \mathcal{T}\left(X^{\star}\right)\right)$. With this and discussions in [59], the left hand side of Eq. (A.4) is rewritten as

$$
\begin{aligned}
& \mathrm{E}\left\{\left(\boldsymbol{\xi}^{*} G \boldsymbol{\xi}\right)^{2}\right\} \\
= & \mathrm{E}\left\{\left(\mathcal{T}(\boldsymbol{\xi})^{\top} \mathcal{T}(G) \mathcal{T}(\boldsymbol{\xi})\right)^{2}\right\} \\
= & \sum_{i, j, k, l} \hat{g}_{i, j} \hat{g}_{k, l} \mathrm{E}\left\{\hat{\xi}_{i} \hat{\xi}_{j} \hat{\xi}_{k} \hat{\xi}_{l}\right\},
\end{aligned}
$$

where $\hat{g}_{i, j}$ and $\hat{\xi}_{k}$ are the $(i, j)$-th element of $\mathcal{T}(G)$ and $k$-th element of $\mathcal{T}(\boldsymbol{\xi})$, respectively. In [60], for $\mathbf{x} \sim \mathcal{N}(\mu, \Sigma)$, the forth moment about the mean has been derived as

$$
\begin{aligned}
& \mathrm{E}\left\{\left(x_{i}-\mu_{i}\right)\left(x_{j}-\mu_{j}\right)\left(x_{k}-\mu_{k}\right)\left(x_{l}-\mu_{l}\right)\right\} \\
= & \sigma_{i, j} \sigma_{k, l}+\sigma_{i, k} \sigma_{j, l}+\sigma_{i, l} \sigma_{j, k},
\end{aligned}
$$

where $x_{i}, \mu_{i}$ and $\sigma_{i, j}$ are the $i$-th element of $\mathbf{x}$, the $i$-th element of $\boldsymbol{\mu}$ and the $(i, j)$-th element of the real valuedcovariance matrix $\Sigma$, respectively. With Eq. (A. 6), Eq. (A.4) is rewritten as

$$
\begin{aligned}
& \mathrm{E}\left\{\left(\boldsymbol{\xi}^{*} G \boldsymbol{\xi}\right)^{2}\right\} \\
= & \frac{1}{4}\left\{\sum_{i, j, k, l} \hat{g}_{i, j} \hat{g}_{k, l}\left({\hat{x^{\star}}}_{i, j}{\hat{x^{\star}}}_{k, l}+{\hat{x^{\star}}}_{i, k}{\hat{x^{\star}}}_{j, l}+\hat{x}_{i, l}{\hat{x^{\star}}}_{j, k}\right)\right\} \\
= & \frac{1}{4}\left\{\operatorname{Tr}\left(\mathcal{T}(G) \mathcal{T}\left(X^{\star}\right)\right)^{2}+2 \operatorname{Tr}\left(\mathcal{T}(G) \mathcal{T}\left(X^{\star}\right) \mathcal{T}(G) \mathcal{T}\left(X^{\star}\right)\right)\right\},
\end{aligned}
$$

where $\hat{x}_{i, j}$ is the $(i, j)$-th element of $\mathcal{T}\left(X^{\star}\right)$. In deriving the above second equality, we have used the property that the matrices $\mathcal{T}(G)$ and $\mathcal{T}\left(X^{\star}\right)$ are symmetric. Let $V$ be a matrix such that $V V^{\top}=\mathcal{T}(G)$, where such a $V$ can be found since $\mathcal{T}(G)$ is a positive semidefinite. With this decomposition, the relations

$$
\begin{aligned}
& \operatorname{Tr}\left(\mathcal{T}(G) \mathcal{T}\left(X^{\star}\right) \mathcal{T}(G) \mathcal{T}\left(X^{\star}\right)\right) \\
= & \operatorname{Tr}\left(V^{\top} \mathcal{T}\left(X^{\star}\right) V \cdot V^{\top} \mathcal{T}\left(X^{\star}\right) V\right) \\
\leq & \operatorname{Tr}\left(V^{\top} \mathcal{T}\left(X^{\star}\right) V\right)^{2} \\
= & \operatorname{Tr}\left(\mathcal{T}\left(X^{\star}\right) \mathcal{T}(G)\right)^{2}
\end{aligned}
$$

are obtained. In the above relations, we have used the properties that the matrix $V^{\top} \mathcal{T}\left(X^{\star}\right) V$ is positive semidefinite and $\operatorname{Tr}(X X) \leq \operatorname{Tr}(X)^{2}$ for any positive semidefinite matrix $X$. In [41], it has been shown that $\operatorname{Tr}(\mathcal{T}(X) \mathcal{T}(Y))=2 \operatorname{Tr}(X Y)$ for positive semidefinite matrices $X$ and $Y$. Combining this result and Eqs. (A- 7) (A· 8), we arrive at the relation

$$
\mathrm{E}\left\{\left(\boldsymbol{\xi}^{*} G \boldsymbol{\xi}\right)^{2}\right\} \leq 3 \operatorname{Tr}\left(G X^{\star}\right)^{2}
$$

This is the desired result.

We have proven Eq. (A. 4) for general $L$. For $L=2$ and $L=4$, we have the same expressions of Eq. (A.4).

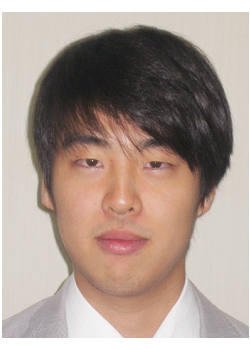

Hirofumi Tsuda received the bachelor of engineering, master of informatics and Ph.D. degrees from Kyoto University, in 2015, 2016 and 2019, respectively. His research interest includes optimizations and communication theories.

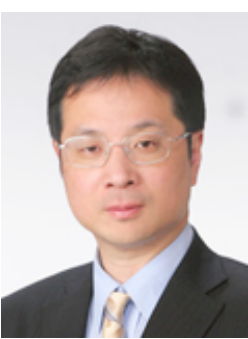

Ken Umeno is Professor at Graduated School of Informatics, Kyoto University. He received his BSc degree in electronic communication from Waseda University, Japan in 1990. He received his M.Sc. and Ph.D. degree in physics from the University of Tokyo, Japan in 1992 and in 1995, respectively. Prior to joining Kyoto University in 2012, he worked for the Ministry of Posts and Telecommunications, Communications Research Laboratory (currently National Institute of Information and Communications Technology of Japan, NICT). From 2004 to 2012, he was the CEO and the president of ChaosWare, Inc, a first spin-off company of NICT as well as a principal investigator of NICT. He received the LSI IP Award in 2003, the Telecom-System Award in 2003 and 2008 respectively. He holds 50 registered Japanese patents and 24 registered US patents in the fields of telecommunications, security, and financial engineering. His research interests include ergodic theory, statistical computing, coding theory, chaos theory and its applications to communications and computing. 